\title{
Dynamic Processes of Social and Economic Interactions: On the Persistence of Inefficiencies
}

\author{
Armando Gomes \\ University of Pennsylvania \\ Philippe Jehiel \\ Paris-Jourdan Sciences Economiques and University College London
}

\begin{abstract}
An economy with a finite number of agents and a finite number of states is considered. An exogenous institutional rule prescribes which moves from one state to another are feasible to each coalition. At each time, an agent is called to act with some exogenous probability, and he chooses a coalition, a feasible new state to move the economy to, and side payments between the agents in the coalition. The setup can be applied to various dynamic processes of social and economic interactions such as legislative bargaining, coalition formation, or exchange economies. Whenever agents are unable to write long-term contracts, but are otherwise unconstrained both in their ability to write arbitrary spot contracts and in their ability to collude, there can be long-run inefficiencies (with cycles or inefficient steady states). However, when agents are sufficiently patient, the initial state from which the process starts plays no role in the long run. Moreover, when there exists an efficient state that is free of negative externalities (in the sense that a move away from that state does not hurt the agents whose consent is not required for the move), the system must converge to
\end{abstract}

We thank Pierre-Andre Chiappori (the editor), two anonymous referees, Leonardo Felli, Richard Kihlstrom, and participants of the Centre for Economic Policy Research Economic Theory Conference (Gerzensee, Switzerland), the North American summer meeting of the Econometric Society (Los Angeles), the National Bureau of Economic Research Decentralization Conference (Washington), the Southeast Economic Theory Conference (Miami), and the conference on Local Public Goods, Politics and Multijurisdictional Economies (Paris). Part of this research was started while Jehiel was visiting the Institute for Advanced Study (Princeton University), the hospitality of which is gratefully acknowledged. Gomes thanks the financial support provided by the Rodney L. White Center for Financial Research.

[Journal of Political Economy, 2005, vol. 113, no. 3]

(C) 2005 by The University of Chicago. All rights reserved. 0022-3808/2005/11303-0006 $\$ 10.00$ 
this efficient state in the long run. It is thus more important to design institutions guaranteeing the existence of an efficient negative externality-free state than to implement a fine initialization of the process.

\section{Introduction}

An economy with a finite number of agents and a finite number of states is considered. When the economy lies in a state, every agent receives a payoff flow that may arbitrarily depend on the identity of the agent and the state. An exogenous institutional rule prescribes how states can be changed over time by specific coalitions of agents. At each time, an agent is called to act with some exogenous (possibly statedependent) probability. He can choose a coalition and a new feasible state to move the economy to. Side payments between agents are allowed, and agents are assumed to be patient and farsighted; specifically, when choosing a move, they take into account how other agents might in turn react, and so on without limit. The setup can be interpreted as providing an abstract model of dynamic processes of social and economic interactions. Three potential applications are discussed: legislative bargaining, coalition formation, and exchange economies. In legislative bargaining, states stand for policies, and the set of allowed transitions is defined by the constitutional setting. In coalition formation, states stand for the partitions of agents into coalitions, and transition from one coalition structure to another requires the consent of those agents who change coalition. Finally, in exchange economies, states stand for the allocations of goods to agents, and the notion of property right dictates that any agent whose allocation is affected should approve the transition.

Our interest lies in the study of the efficiency and convergence properties of such dynamic processes of social and economic interactions. The contracting possibilities available to agents are clearly an important determinant of the outcome of the interactions among agents. Specifically, if long-term contracts can be offered, then efficiency obtains, as should be expected from the Coase theorem. That is, by allowing commitments to actions or nonactions to be taken in future stages, longterm contracts guarantee that the economy moves immediately to the Pareto-efficient state with no further move. More surprisingly, we establish that if only spot contracts can be offered-that is, only commitments to immediate actions are available-efficiency also obtains in the dynamic economy, and there is also an immediate move to the efficient state with no further move. In the spot contracting scenario, efficiency obtains because the designer of the contract has the possibility of threatening any agent included in the contract to make the worst possible 
move for the agent, in case of disagreement with this agent, thus inducing the agent to make a payment to the designer just to stay in the efficient state. ${ }^{1}$ But, when the approached agents can react to the proposed spot contract by forming a ring (thereby coordinating their response), the picture is quite different. ${ }^{2}$ Indeed, most threats targeted to deter deviations by single agents then become ineffective, since by jointly refusing the contract, the approached agents can always force the proposer to withdraw his offer. We show that if a solo player has no right to change the state of the economy on his own, then the equilibrium contract offers have a simple form (which applies to every state and to whoever is the proposer): take-it-or-leave-it offers conditioned on the acceptance of all approached agents, where no change of state and no transfers take place if one or more approached agents refuse the offer.

We believe that there are quite a few applications for which the restriction to collusion-proof spot contracts is very reasonable. The main goal of this paper is to study the equilibrium dynamics induced by such a collusion-proof spot contracting scenario (which is equivalent to the simple take-it-or-leave-it offer contracting scenario described above). In contrast to our other contracting scenarios, we observe that rich dynamics and long-run inefficiencies may arise. More precisely, we observe in a series of examples that the economy may sometimes have cycles (i.e., the economy keeps moving from one state to another even in the long run), and when it converges, the limiting states may sometimes be efficient and sometimes inefficient. Whether or not the economy converges to a stable state may also depend on the probabilities that the various agents are selected to make proposals. Thus efficiency is no longer guaranteed, and we provide several examples taken from our three main applications-legislative bargaining, coalition formation, and exchange economy-in which this is the case.

Of course, whether or not inefficiencies arise depends on the form of payoffs and the set of allowed transitions as well as the discount factor and the probabilities of being proposer in every state. An important contribution of this paper is to provide conditions under which efficiency should be expected. The key intuition (to be further analyzed and refined) for why inefficiencies and instabilities arise is that sometimes a move by agents to an inefficient state may enhance their bargaining position as a result of negative externalities imposed on other agents.

\footnotetext{
${ }^{1}$ This kind of blackmailing contract has been first highlighted in static contracting setups by Jehiel, Moldovanu, and Stacchetti (1996). We use its property to establish efficiency in our dynamic game.

${ }^{2}$ In some sense, this amounts to giving the same commitment abilities to the designer as to the approached agents.
} 
Several results provide a sharp characterization of the efficiency and convergence properties of equilibria. A robust conclusion that applies to any specification is that there is no effect of the initial state on the long-run properties of the system, as long as agents are sufficiently patient. Observe that this insight holds true whether the system cycles or converges and whatever the efficiency properties of the limit behavior of the system. The irrelevance of the initial state with respect to the long-run properties indicates that there is no point in reinitializing the system to another state if one is to leave the rest of the process unchanged.

Since inefficiencies may sometimes occur and since efficiency is affected by the institutional transition rules, we may conclude that reforms aimed at improving economic efficiency should mostly bear on the form of the allowed transitions (rather than on a fine initialization of the system). Whenever possible, reforms should also bear on the contracting possibilities available to agents.

When can we guarantee that the equilibria of our economy are efficient and eventually converge to a steady state? The answers to these questions are of importance to assess or improve the economic performance of existing institutions. We identify a necessary and sufficient condition that guarantees for patient agents the convergence to an efficient state irrespective of the probabilities that the various agents are selected to make proposals (and irrespective of the initial state, as implied by the previous result). That condition combines properties of the allowed transitions and of the flows of payoffs obtained by the agents in the various states. In short, the condition amounts to the existence of an efficient state that is free of negative externalities in the sense that if a (possibly indirect) transition from that efficient state to another state is possible without the consent of some agent, then this agent is no worse off (in terms of immediate flows of payoffs) in the original (efficient) state than in the reachable state.

The existence of an efficient negative externality-free (ENF) state is key for the following reason. Consider an efficient state $a$ that is not free of negative externalities. That is, there is a group $S$, a state $b$ such that $S$ can move from $a$ to $b$ (possibly in several steps), and an agent $i$ outside $S$ who derives a higher flow of payoff in state $a$ than in state $b$. There is always the temptation for group $S$ to move from state $a$ to state $b$ in order to extract some surplus from agent $i$ (in exchange for the equilibrium prospect of moving back to state $a$ ). This effect indeed destabilizes state $a$ whenever the probability that agent $i$ is the proposer at state $b$ is sufficiently small. In contrast, when the efficient state $a$ is negative externality-free, no such move can destabilize $a$, and the system must converge to state $a$ in a finite number of steps whatever the prob- 
abilities that the various agents are selected to make proposals in the various states and whatever the initial state.

Our insights have several implications that we now review. Some implications echo or complement results already present in the literature (although typically obtained in less general setups). Others shed new light on strands of literature that used different (generally static) approaches.

In legislative bargaining, a legislator cannot credibly commit to actions to be taken in future legislatures, so ruling out long-term contracts seems like the natural assumption. Simple majority procedures for implementing changes of policy do not, in general, guarantee the existence of an ENF state even in those contexts in which one policy is preferred to any other policy by a majority (see the voting example in Sec. III.A). Received voting theory based on static approaches would predict in such a case that a policy winning against any other policy-a so-called Condorcet winner-should emerge. However, in our dynamic setup, cycles between policies may arise at equilibrium because of the nonexistence of an ENF state. It should be noted that in contrast to static approaches (à la Condorcet, say), there is no problem in our dynamic setting with speaking of cycles. And our insights (about the emergence of cycles in dynamic settings) may suggest a new reason for political instability in democracies. Besides, our theory suggests that in order to guarantee convergence to an efficient state irrespective of who sets the agenda, unanimity constraints may have to be imposed (at least to leave the efficient state since this constraint guarantees the existence of an ENF state). Or, if unanimity constraints cannot be imposed, it is important to adjust finely the probabilities of who sets the agenda in every state of the world in order to improve the functioning of the legislative process. ${ }^{3}$

Our model also delivers interesting insights into exchange economies with durable goods or assets. When there are no externalities (i.e., when agents care only about their allocation), the existence of ENF states is guaranteed, and thus convergence to an efficient state follows (this complements results obtained by Gale [1986] in a setting with nondurable goods and no discounting). When there are externalities (i.e., when agents care about the entire profile of allocations; see Jehiel and Moldovanu [1995a, 1995b]), long-run inefficiencies may arise (because

\footnotetext{
${ }^{3}$ Of course, we are not advocating in favor of unanimity constraints, which in a number of cases (in particular involving voting imperfections) may be very inefficient. But our analysis highlights some potential problems with other less demanding majority requirements.
} 
there need not be an ENF state). In simple instances, though, efficiency might obtain even with externalities (as long as an ENF state exists). ${ }^{4}$

Our setup can also be used to speak of coalition formation in a new way. In previous works, the concept of core played an important role in the analysis of stability. Our analysis sheds new light on the issue of stability (or convergence) by identifying a new concept (other than the core), that is, the concept of ENF states, which appears to be the key stability concept with farsighted patient agents (whereas the core is more useful for myopic agents).

The rest of the paper is organized as follows. In Section II we describe the model and review the related literature. In Section III we explain how the model can be used in a variety of applications. In Section IV we provide a contract-theoretic motivation for the model. In Section V we derive a number of general results about the efficiency and convergence properties of the equilibria. Concluding remarks appear in Section VI. All proofs are gathered in the Appendix.

\section{The Model}

Consider an economy with a finite number $n$ of agents, a finite number $m$ of possible states, and infinitely many periods of interactions, $k=$ $1,2, \ldots$. We let $N=\{1, \ldots, n\}$ denote the set of agents and $Z$ denote the set of states. Agents all have the same discount factor $\delta,{ }^{5}$ which in most of the paper will be assumed to be close to one. As the economy lies in state $a \in Z$, agent $i$ derives a flow of utility equal to $(1-\delta) v_{i}(a)$ per period. ${ }^{6}$ Side payments between agents can also be made at any period and in any state, and utilities are assumed to be transferable between agents (i.e., quasi-linear in money). ${ }^{7}$ That is, let $a^{k}$ and $t_{i}^{k}$ be,

\footnotetext{
${ }^{4}$ For example, suppose that there is only one good consisting of a cost-reducing technology and the agents are firms competing in an imperfectly competitive fashion. Suppose that the efficient state (the one that maximizes the profit of all firms) requires that firm $i$ gets the innovation and suppose that all other firms' profits are higher when firm $i$ gets the innovation (because, say, firm $i$ induces a softer competition than other firms). Then our result shows that firm $i$ will eventually own the cost-reducing innovation and will never resell it to another firm (because the efficient state-firm $i$ owning the good-is negative externality-free).

${ }^{5}$ The analysis easily extends to the case in which agents have different discount factors. We have chosen to present the model with equally patient agents to alleviate the notation a bit.

${ }^{6}$ Thus if the state were to be $a$ permanently, agent $i$ would derive a total utility equal to $v_{i}(a)$.

${ }^{7}$ The transferability assumption implicitly rules out capital market imperfections, in particular related to borrowing constraints (where the budget of an agent is insufficient to finance some desired side payment). Note that if the payoffs are normalized so that all $v_{i}(a) \geq 0$, then a sufficient condition for agents not to be budget constrained (so that capital market imperfections become irrelevant) is that each agent $i$ has wealth $w_{i} \geq$ $\max _{a \in Z}\left[\sum_{j \in N} v_{j}(a)\right]$.
} 
respectively, the state in period $k$ and the transfer received by agent $i$ in period $k$. Let $\tilde{\zeta}$ denote the stochastic process governing $a^{k}$ and $t_{i}^{k}$. Agent $i$ 's expected utility induced by this stochastic process is

$$
E_{\hat{\zeta}}\left[\sum_{k=0}^{\infty} \delta^{k}\left[t_{i}^{k}+(1-\delta) v_{i}\left(a^{k}\right)\right]\right],
$$

where $E$ denotes the expectation operator.

A key feature of our analysis is that the transitions from states to states are determined by the agents themselves in every period. In every state, agents are selected to make proposals. A proposal consists of an offer to a group of agents to move from the current state to another statewhere the move is required to be feasible for the group-and possibly side payments between the group members.

The set of transitions that a group of agents is allowed to implement depends on the institutional setting. In the next section we consider several major applications of our setup, which in each case suggest a natural way of defining the set of allowed transitions. For example, in exchange economies, the states stand for the various possible allocations of the goods, and the change of ownership of an asset requires the approval of the buyer and the seller of the asset, which in turn defines the set of transitions that a group of agents can implement (i.e., any change of ownership of the assets owned by the group members, but not of assets that are not owned by them).

The institutional setting is taken as given in our paper. Accordingly, the set of allowed transitions is given exogenously. Formally, the set of allowed transitions is defined as a relation $\rightarrow$ over pairs of states $(a, b)$ and groups $S$ of agents, where $a \rightarrow_{S} b$ means that if all agents in $S$ approve a transition from state $a$ to state $b$, the transition can be implemented. ${ }^{9}$

Note that, for example, in the voting application to be discussed below, the states stand for the policies or laws, and any majority of agents is enough to implement a change of policy. As will be further discussed in the next section, this is covered by our model by letting $a \rightarrow_{s} b$ whenever $S$ contains a majority of agents, $|S|>n / 2$. Thus changes of state do not require, in general, the unanimous consent of a specific (or pivotal) group $S$ of agents to be implemented.

Throughout the paper, we make the following assumptions about the transition relation.

Assumption 1. For all $a \in Z$ and $S \subseteq N, a \rightarrow_{S} a$. (Staying in the same state is always possible.)

\footnotetext{
${ }^{8}$ Later on this stochastic process will be made endogenous (the result of the strategies employed by the agents).

${ }^{9}$ Alternatively, the transition rule is associated with a subset $\mathcal{F} \subset Z \times Z \times 2^{N}$, so that $a \rightarrow_{S} b$ if and only if $(a, b, S) \in \mathcal{F}$.
} 
Assumption 2. For all $a, b \in Z$, if $a \rightarrow_{S} b$, then $a \rightarrow_{T} b$ whenever $S \subset T$. (If a subcoalition $S \subset T$ may move from state $a$ to state $b$, so may coalition $T$.)

Assumption 3. For all $a, b \in Z, a \rightarrow_{N} b$. (If everybody agrees, a transition from state $a$ to state $b$ is always possible.)

In words, assumption 1 states that one may always (if one wishes) stay in the current state (the status quo is always available). Assumption 2 states that if the consent of agents in $S$ is enough to move from state $a$ to state $b$, then the extra consent of agents outside $S$ cannot make the move unfeasible. Assumption 3 states that if everybody approves the change from $a$ to $b$, then it can be done. In all applications discussed below, assumptions 1-3 are trivially met.

The timing of events in our game is as follows. Let $a$ be the current state at the start of period $k$. Agent $i$ is selected with probability $p_{i}(a)$ to make a proposal at that state. When selected, agent $i$ 's offer $\tau$ consists of a subset $S$ of agents (with $i \in S$ ), a state $b$, such that $a \rightarrow_{S} b$, and transfers $t=\left(t_{j}\right)_{j \in S}$ between members of $S$ such that $\sum_{j \in S} t_{j}=0$. In words, the offer $\tau=(S, b, t)$ stands for a proposal made by agent $i$ to agents $j \in S$ to switch from state $a$ to state $b$ in exchange for side payments $t=\left(t_{j}\right)_{j \in S}$, where $t_{j}$ is the payment received by agent $j$.

When the offer $\tau=(S, b, t)$ is made, the agents in $S$ respond to the offer by yes or no. ${ }^{10}$ If the offer is rejected by any agent, then the economy moves to the next period and the state remains unchanged (i.e., it remains $a$ ), with no side payments being made. Otherwise, if all agents in $S$ accept the offer, then the state of the economy moves immediately to $b$ and the (lump-sum) transfers $t$ are made. ${ }^{11}$ So offers in the model are take-it-or-leave-it offers in which any approached agent can block its implementation.

The above dynamic game is characterized by the transition relation $\rightarrow$, the flows of payoffs in every state $v=\left\{v_{i}(a)\right\}_{i, a}$, the probabilities of being proposer in every state $p=\left\{p_{i}(a)\right\}_{i, a}$ and the discount factor $\delta$. This game is referred to as the economy $\mathcal{E}(\rightarrow, v, p, \delta)$. Some properties will refer to the specification of the transition relation $\rightarrow, v$, and $p$ only (but not $\delta)$; we shall then speak of the $(\rightarrow, v, p)$ economy and denote it by $\mathcal{E}(\rightarrow, v, p)$. Some properties will refer to the transition relation and

\footnotetext{
${ }^{10}$ We could assume either that agents respond in order (the specific order being irrelevant) or that they respond simultaneously, in which case we restrict attention to equilibria in undominated strategies (so as to avoid coordination problems).

${ }^{11}$ Note that the offer is made at the start of a period. Thus if a transition is implemented, it takes place immediately (there is no lag in the implementation).
} 
$v$ (but not $p$ and $\delta$ ); we shall then speak of the $(\rightarrow, v)$ economy and denote it by $\mathcal{E}(\rightarrow, v){ }^{12}$

Equilibrium and efficiency.-Throughout the paper, we restrict attention to Markov perfect equilibria (MPE), which we refer to as equilibria in the text. In an MPE, the strategies used by the players may depend only on the current state of the economy (and also for the proposer on his identity and for the responders on the proposal). Mixed strategies that take the form of randomizations over proposed offers are allowed and in fact required to guarantee the existence of MPE. ${ }^{13}$

Formally, a Markovian strategy for player $i$ denoted by $\sigma_{i}$ specifies for every state $a$ such that $p_{i}(a)>0$ a probability distribution over all feasible offers $(S, b, t)$ that agent $i$ can possibly make at state $a$ (i.e., satisfying $\left.a \rightarrow_{s} b\right)$, and for every state $a^{\prime}$ and every offer $\left(S^{\prime}, b^{\prime}, t^{\prime}\right)$ such that $i \in$ $S^{\prime}$ and $a^{\prime} \rightarrow_{S^{\prime}} b^{\prime}$, a probability of acceptance for player $i$.

Definition 1. Markov perfect equilibrium.-A strategy profile $\sigma=$ $\left(\sigma_{i}\right)_{i=1}^{n}$ is an MPE if, for each player $i, \sigma_{i}$ is a Markovian strategy, and after every history of play, $\sigma_{i}$ is a best response for player $i$ when other players $-i$ play according to $\sigma_{-i}$.

Efficiency in our context boils down to welfare efficiency, since utilities are assumed to be transferable. Accordingly, we have the following definition.

Definition 2. Pareto efficiency.-A state $a \in Z$ is efficient if $a \in$ $\arg \max _{a^{\prime} \in Z} \sum_{i=1}^{n} v_{i}\left(a^{\prime}\right)$.

In order to interpret our results more broadly, we shall consider the following extra assumption on the transition relation.

Assumption 4. For all $i$, there are no $a, b \in Z, a \neq b$, such that $a \rightarrow_{i i\}} b$. (A single player $i$ alone cannot change the state.)

In words, assumption 4 states that a single player alone cannot change the state of the economy. Even though assumption 4 is not needed for the analysis of the model defined above, we note that the assumption is met in most of the applications to be discussed below. Besides, assumption 4 will allow us to interpret our dynamic game as a game in which any collusion-proof spot contract can be offered by the proposer,

\footnotetext{
${ }^{12}$ The bargaining friction considered in our model is that players are impatient and bargaining takes place in real time so that players derive flows of payoffs as the bargaining process goes on. An alternative friction considered in the literature is the risk of breakdown after each round of negotiation. If breakdown is assumed to result in the stopping of the economy at the current state with no further interaction (i.e., if the final state is $a$, player $i$ derives a payoff equal to $\left.v_{i}(a)\right)$, it is readily verified that under the von NeumannMorgenstern assumptions, the economy with exogenous risk of breakdown and the one with discounting and flows of payoffs are equivalent and thus have the same equilibria (see also Binmore, Rubinstein, and Wolinsky 1986).

${ }^{13}$ Mixed strategies may as usual be interpreted as pure strategies by enriching the model and allowing the players to adjust their behavior on a signal that they are the only ones to observe (e.g., an idiosyncratic one-shot perturbation of the flow of payoffs $v_{i}(a)$ ). This is Harsanyi's purification idea.
} 
thus a priori allowing for more elaborate offer contracts than the above model permits (see Sec. IV). Thus, under assumption 4 (and the collusion-proofness requirement), the only contractual constraint imposed by our model is that only spot contracts can be offered (i.e., no longterm contracts can be proposed).

Related literature.-The noncooperative model used in this paper shares a number of features with the model used by Gomes (forthcoming) to study coalitional bargaining problems with externalities. The states of the economy in his setting are the contracts written by coalitions (which can only expand), and he shows that equilibria always exist and are efficient if the grand coalition is efficient and players are patient. ${ }^{14}$ However, the framework used in our paper differs from Gomes's in a key dimension: we allow for more general transition relations that include the possibility that coalitions may break up, whereas in Gomes's article, coalitions can only expand and become coarser. ${ }^{15}$ Allowing for such general transition rules is indispensable to be able to generate cycles such as those arising in the examples of Section III and to analyze the conditions that rule out cycles and inefficiencies (Sec. V).

There are a few other papers in the bargaining literature that share with the present paper the generality of the setup. They include Rosenthal's (1972) "effectiveness form," Greenberg's (1990) "inducement correspondence," and more recently those by Chwe (1994), Xue (1998), and Konishi and Ray (2003), who all use specifications similar to our transition relation. Konishi and Ray also model the process of coalition formation in real time with agents receiving a flow of payoffs. An important distinction between our approach and the one in these studies is that in our setup the only decision makers are the agents themselves (i.e., our approach is purely noncooperative) whereas the other mentioned approaches include fictitious bodies (standing for the coalitions) as the decision makers. Moreover, we allow for side payments between agents and we impose some mild restrictions on the allowed transitions (which are satisfied in all applications we discuss). That extra structure of our setup allows us to obtain sharp predictions in terms of the effect of the initial state and about the conditions ensuring convergence to an efficient state, which the previous literature did not obtain.

\footnotetext{
${ }^{14}$ In our setting too, when the grand coalition is efficient, it is negative externality-free and efficiency must obtain (see proposition 6 and the discussion thereafter).

${ }^{15}$ It should also be mentioned that we consider only economies with finite state spaces, whereas Gomes (forthcoming) allows for infinite state space, which arises naturally in the context of coalitional bargaining games with externalities in which players can offer payoff contracts (see also Sec. III. $B$ for further discussion of coalitional bargaining games).
} 


\section{Applications}

In this section we review a number of applications to which our general model is relevant. For each application, we discuss the meaning of the main assumptions of the model. In particular, we review the meaning that (i) the transition relation satisfies assumptions 1-4, (ii) only spot contracts can be proposed, and (iii) payoffs are flows occurring during the bargaining process. We also exhibit in each case an illustrative example with rich equilibrium dynamics, and we discuss how our general insights (to be developed later) help to understand the application.

\section{A. Legislative Bargaining}

Our first main application is legislative bargaining. Consider a situation with $n$ legislators, $i=1, \ldots, n$, in which there may be ongoing transitions from policies to policies in a set $Z$. Policies can be changed whenever a new parliamentary session comes about. The time separating two sessions corresponds to one period in our model. Clearly, the current policy $a \in Z$ (which prevails between two sessions) affects the flow of payoffs received by the legislators through the consequences that the policy may have on the legislators (the consequences may depend on how the constituency represented by the legislator appreciates the policy). Hence the flow formulation is natural in this application, and we let $v_{i}(a)$ be the normalized flow of payoff received by legislator $i$ when policy $a$ prevails. Besides, the current policy may also have an effect on who has the control over the agenda setting. Accordingly, we let $p_{i}(a)$ be the probability that legislator $i$ has the control in state $a$. Regarding the transition relation $\rightarrow$, this is clearly defined by the rules of the constitution. For example, a constitution specifying that any change of policy can be implemented provided that a strict majority of legislators approves it can be represented as $a \rightarrow_{S} b$ whenever the number of members of $S$ (i.e., $|S|)$ represents a (strict) majority of $N$ (i.e., $|S|>n / 2$ ). Any other qualified majority rule can be represented similarly. ${ }^{16}$ Clearly, any such transition relation satisfies assumptions 1-3. Assumption 4 is also satisfied provided that the consent of at least two legislators is required to change any policy (which is obviously the case in qualified majority rules except for dictatorship).

Our model of legislative bargaining implicitly assumes that if a majority $S$ proposes a switch from policy $a$ to policy $b$, this is implemented. In a number of legislative bargaining contexts, though, a policy is not

\footnotetext{
${ }^{16}$ If a majority can impose taxation on a minority, this can be represented within our setup by expanding the set of policies to specify different taxation scenarios, still keeping the assumption that side payments (outside the direct effects of the state on the payoff that are captured by $\left.v_{i}(a)\right)$ take place only among the agents who approve the transition.
} 
implemented through the initiative of a majority. A proposal to vote on policy $b$ is put on the agenda (by whoever controls the agenda setting), and a vote among all legislators takes place. If a majority votes in favor of it, policy $b$ is implemented, but not otherwise.

Our model also fits with such a setup, but one needs to think of the agenda setter as being able to implement a bribing game prior to the effective vote. (This is close in spirit to the distributive approach first proposed by Shepsle [1979] and further developed by Baron and Ferejohn [1989] and Diermeier and Myerson [1999].) The offer $(S, b, t)$ made by proposer $i$ in our model should be interpreted as follows: Legislator $i$ buys the votes (for $b$ ) of legislators $j \neq i$ in $S$ in exchange for the bribes defined by the transfers $t{ }^{17}$ The bribes and sales of voting rights are all made contingent on the acceptance of the deal by all agents in $S$. Otherwise, the status quo (i.e., current policy a) prevails, with no bribe being made ${ }^{18}$ It is not difficult to see that such a legislative bargaining game is formally equivalent to assuming in our model that $a \rightarrow_{S} b$ whenever $|S|>n / 2$.

Baron and Ferejohn (1989) have proposed a model of legislative voting that resembles the one considered here with the notable difference that once a policy $a$ is implemented, this is the end (see also Banks and Duggan 2001). By contrast, our setting allows the legislators to go back and forth from one policy to another. This possibility, which seems consistent with common practice, will in turn give rise to rich dynamics, as will be illustrated later.

We now discuss two features of our model in the legislative bargaining context: the possibility of side payments and the restriction to spot contracts.

The possibility of side payments.-When legislators represent different bodies of a government, side payments can be interpreted as budgetary transfers between the various bodies. In some circumstances, though, explicit side payments between legislators seem harder. However, even if explicit side payments are not possible, they can often be viewed as reduced forms for favors that legislators would make between themselves (see also Diermeier and Merlo 2000). Our model considers the benchmark case in which such favors are a perfect substitute for side payments. ${ }^{19}$

${ }^{17}$ They may have either sign in our model (see below).

${ }^{18}$ This implicitly assumes that the legislators in $S$ can first observe whether everyone has approved the deal before the sale of voting rights and bribes are implemented. Also, our restriction to spot contracts should be understood as allowing for any kind of commitment within the parliamentary session.

${ }^{19}$ As we shall see, even if side payments are feasible, inefficiencies must arise in some cases. A fortiori, inefficiencies would also arise when side payments are restricted or favors are not a perfect substitute for side payments (even though in some cases, such as our voting example in Sec. III, the impossibility of side payments may help). The exact analysis 
Restriction to spot contracts. - In legislative bargaining contexts, there seem to be very few examples of long-term contracts. (In most developed countries, a legislator would have a very hard time trying to credibly commit to actions to be taken in future legislatures.) ${ }^{20}$ Thus assuming that only spot contracts can be proposed seems plausible.

To illustrate the predictions of our model in the legislative bargaining context, consider the following voting game with three players $i=1$, 2 , and 3 and four possible policies $s=a, b, c$, and $d$, with corresponding flows of payoffs as shown below:

\begin{tabular}{lllll}
\hline \hline$v_{i}(s)$ & $a$ & $b$ & \multicolumn{1}{c}{$c$} & $d$ \\
\hline 1 & 1.5 & 1 & 0 & 1.6 \\
2 & 1 & 0 & 1.5 & 1.6 \\
3 & 0 & 1.5 & 1 & 1.6 \\
\hline
\end{tabular}

Any policy can be passed by majority voting. We assume that all players can be the proposers with equal probability in all states.

Any static voting approach would predict that policy $d$ should emerge, since no majority prefers $a$, $b$, or $c$ over $d .^{21}$ In fact, policy $d$ is even unanimously preferred over $a, b$, and $c$. Accordingly, for low discount rates the equilibria of our dynamic voting game are such that from any state there is a move to state $d$ and the economy remains at $d$ forever. ${ }^{22}$

But the picture changes drastically when players get more patient. Staying in $d$ forever is no longer part of an equilibrium, and cycles must emerge in equilibrium when players are patient enough.

To get an intuition, we first observe that an immediate jump to state $d$ without any further move (which would mimic the equilibrium pattern in the myopic case) cannot be part of an equilibrium. Indeed, if such transitions were part of an equilibrium, at state $d$ players would strictly

of when efficiency can be expected in the nontransferable utility case would, however, require further research.

${ }^{20}$ While it is clearly beyond the scope of this paper to analyze why long-term contracts are so rare in political contexts (changes in constitutions are one of the very few examples), one may mention a simple argument. In the real world, legislators do not stay in office forever, and even if they do stay in office for a few legislatures, it is hardly conceivable that they could commit to some political actions to be taken next when there is some uncertainty as to whether they will still be in office. The possibility that the legislator may physically change from one period to another may be responsible for why essentially only spot contracts are observed in the political arena. From a formal viewpoint, our model does not assume that legislators change from one period to another. Yet it can easily be reinterpreted this way. In each period $k$ there should be a different player $(i, k)$, where all players $(i, k)$ share the same objective (which can be interpreted as the well-being of the constituency in jurisdiction $i$ ). Clearly, the MPE of our game are also MPE of the multiplayer version of the game and vice versa.

${ }^{21}$ Policy $d$ would be referred to as a Condorcet winner in the political science literature.

${ }^{22}$ The equilibrium values are $\phi_{i}(s)=v_{i}(s)+(2.3 / 3)$ for $s=a, b$, and $c$, which is the status quo plus a third of the surplus, and $\phi_{i}(d)=1.6$. The equilibrium holds for all $\delta<0.46$. 


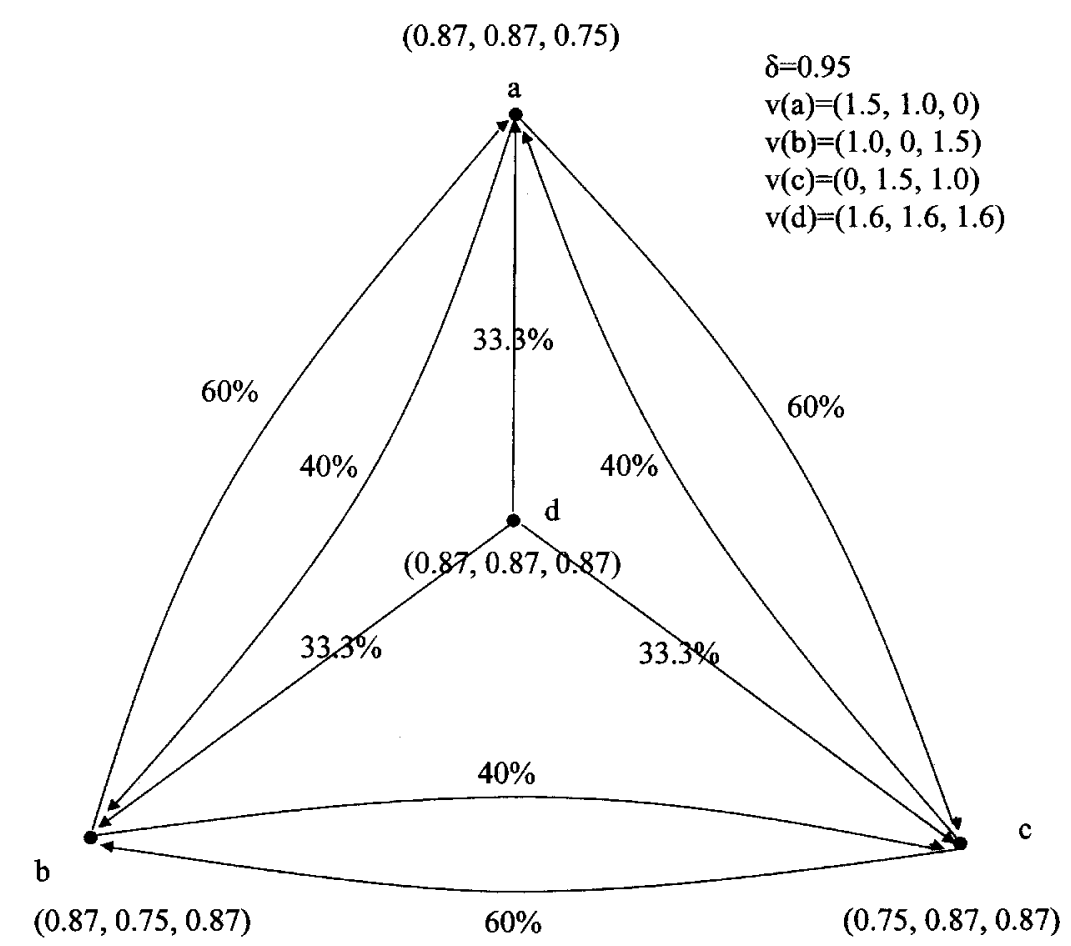

Fig. 1.-Voting game equilibrium values (in parentheses) and transition probabilities (percentages).

prefer moving to another state rather than staying at state $d$, thus leading to a contradiction. To see the point, assume that player 1 is selected to make a proposal at state $d$. When $\delta$ is sufficiently close to one, player 1 would strictly prefer proposing to player 2 to move to state $a$ rather than stay in state $d$. Indeed, a jump to state $a$ induces an immediate loss to players 1 and 2 of $(1-\delta)(3.2-2.5)$. But this immediate loss is more than compensated by the expected gain that players 1 and 2 can make by extracting money from player 3 in exchange for going back to state $d$. Specifically, under the assumed equilibrium transitions, the expected total utility of players 1 and 2 at state $a$ should be $2.5+$ $\frac{2}{3}(4.8-2.5)>3.2$, where 2.5 is the status quo value of players 1 and 2 and $\frac{2}{3}$ is the probability that they can be proposers (thereby extracting the full total state $d$ surplus for them). Hence, when $\delta$ is sufficiently close to one, the expected gain to be made next by players 1 and 2 when at state $a$ more than compensates the immediate loss due to a move to state $a$.

The equilibrium for $\delta=0.95$ is shown in figure 1. It exhibits some 
interesting properties. In equilibrium (see also the Appendix for a formal derivation of the equilibrium), when at, say, state $a$, player 2 strictly prefers moving to state $c$, which is the state that hurts player 1 the most in the short term (payoffs at state $a$ are 1.5, 1, and 0 and at state $c$ are $0,1.5$, and 1 ); player 1 strictly prefers moving to state $b$, which is the state that hurts player 2 the most (payoffs at $b$ are 1,0 , and 1.5); and player 3 is indifferent between moving to state $b$ with player 1 and moving to state $c$ with player 2 . When at state $d$, players 1,2 , and 3 propose to move, respectively, to states $a, c$, and $b$. So whatever the initial state, the system cycles between states $a, b$, and $c$ with an equal frequency of visits for each of these three states; and when players are patient enough, the system never actually visits state $d$.

At first sight it might seem strange that state $d$-which is unanimously preferred to other states-happens to be highly unstable. The reason is that somehow each player can improve his bargaining position by moving to another state, say state $a$ for player 1 . But player 1 alone is not allowed to move to state $a$. Yet, players 1 and 2 together can move to state $a$, and they are jointly in a better bargaining position in state $a$ than in state $d$, since state $a$ allows them to extract surplus from player 3 , who suffers a lot in state $a$. It is interesting to note that in equilibrium, once in state $a$, the system does not move back to state $d$ because players 1 and 2 always prefer to move to either state $b$ or $c$ in order to extract more (in the future) from the left-aside agent (they are also afraid that in state $d$ player 3 might exploit them when he is the proposer).

The fundamental reason why the outcome of the equilibrium is inefficient will be identified formally in the rest of the paper. In short, inefficiencies arise because moving away from the efficient state $d$ to state $a$ can be done without the permission of player 3 , and player 3 is worse off in state $a$ than in state $d$. In the language of our paper, we shall say that the efficient state $d$ is not negative externality-free, and whenever the efficient state is not negative externality-free, inefficiencies may occur when players are patient enough.

It should be noted that if in the same example we had required that to move away from state $d$ unanimous consent were required, then the efficient state $d$ would have been negative externality-free, and the economy would have converged to the efficient state in a finite number of steps (hence no cycles would have occurred). Different transition relations thus result in very different dynamics, and an important contribution of this paper is to identify the conditions on the transition relation that guarantee convergence to an efficient state. ${ }^{23}$

\footnotetext{
${ }^{23}$ Clearly, our results have no counterpart in the static approaches to voting in which the possibility of cycles or instability is solely attributed to the form of payoffs (think of Condorcet cycles).
} 


\section{B. Coalitional Bargaining Games}

Our second main application is dynamic coalition formation. In this application, the set of states can be viewed as the set of partitions $\pi$ of the players $N=\{1, \ldots, n\}$ (we let $Z=\Pi$ be the set of partitions of the $N$ players). Regarding the transition rule, we assume that players who change coalitions should approve the transition. Formally, the transition rule is defined as follows: for any two partitions (states) $\pi$ and $\pi^{\prime}, \pi \rightarrow_{S}$ $\pi^{\prime}$ if and only if $S$ contains those agents whose coalitions changed.

Note that the transition relation clearly satisfies assumptions $1-4$. Besides, it allows for coalitions to break up or expand at any point in time, which is in contrast to most of the literature on noncooperative models of coalition formation. ${ }^{24}$

To illustrate the transition rule, consider a situation with three players $N=\{1,2,3\}$, and assume that the current state is defined by a twoplayer coalition made of players 1 and 2 and a solo coalition made of player 3. We refer to such a partition as [12][3]..$^{25}$ A move from [12][3] to [1][2][3] (which corresponds to a breakup of coalition [12]) can be done with the sole consent of players 1 and 2 (so neither player 1 nor 2 can leave the coalition [12] without the consent of the other player). A move from [12][3] to state [123] (expansion of coalition [12]) or a move to state [13][2] (reorganization of coalitions) requires the consent of all three agents. Also, a move from the grand coalition [123] to any other coalition structure (breakup of the grand coalition) requires the consent of all three agents.

Finally, $v_{i}(\pi)$ is the flow of payoffs obtained by player $i$ when the coalition structure is given by $\pi$. And we let $\delta$ be the discount factor and $p_{i}(\pi) \geq 0$ (such that $\left.\sum_{i \in N} p_{i}(\pi)=1\right)$ denote the probability that agent $i$ makes a proposal in state $\pi$.

The main restriction imposed by our setup is that players cannot commit to future actions, that is, decisions to be made in future stages about whether to stay in or quit coalitions. This, in particular, rules out the possibility for a player to delegate his decision rights (about whether to join or quit a coalition) to another player. But, in many coalition bargaining contexts, the no-commitment assumption sounds plausible.

Related literature.-Previously studied coalition formation games in dynamic settings take either the form of coalitional bargaining models with externalities (e.g., Bloch 1996; Ray and Vohra 1999; Gomes, forthcoming) or the traditional form without externalities (e.g., Gul 1989;

\footnotetext{
${ }^{24}$ Most of the literature assumes that coalitions leave the game when they form (see Chatterjee et al. 1993; Ray and Vohra 1999) or assumes that coalitions can only expand (Gomes, forthcoming).

${ }^{25}$ More generally, we represent coalitions and partitions using brackets in a similar fashion.
} 
Perry and Reny 1994; Moldovanu and Winter 1995; Hart and Mas-Colell 1996). Generally, the externalities of the coalitional game among $N=\{1, \ldots, n\}$ players are described by a partition function $v(S, \pi) \in$ $R$ that stands for the value of coalition $S$ given the partition $\pi=\left\{S_{1}\right.$, $\ldots, S_{k}$ \} of the $N$ players. When there are no externalities (or, in the language of cooperative game theory, when the game has a characteristic form representation), the additional restriction that $v(S, \pi)=v\left(S, \pi^{\prime}\right)$ for all $S \in \pi \cap \pi^{\prime}$ is imposed; that is, the value of coalition $S$ does not depend on the whole architecture of coalitions, but solely on who is in $S$. In our noncooperative model, we specify an arbitrary payoff $v_{i}(\pi)$ compatible with $v(S, \pi)$, that is, any choice satisfying $v(S, \pi)=$ $\sum_{i \in S} v_{i}(\pi)$. However, our approach of choosing an arbitrary payoff is justified because, as in Gomes's (forthcoming) setup, the equilibrium of the game in which the payoffs are endogenous is essentially the same (similar transition probabilities and value function) as the equilibrium of the game in which we fix any arbitrary payoffs.

Our analysis delivers several insights. First, in the traditional situation in which individuals solely care about the composition of the coalition they belong to (i.e., there are no externalities), convergence to the efficient partition is guaranteed (whether it is the grand coalition or not). Second, if the grand coalition is the Pareto-efficient allocation, then it will eventually arise in a finite number of periods, whether or not there are externalities. These two results follow from the observations that in these two cases the efficient state is negative externalityfree (see below for a formal general definition).

But, in more general situations the equilibrium may or may not be efficient depending on whether or not there exists an efficient partition that is negative externality-free. To illustrate the possibility of inefficiency (and rich dynamics), consider the following example:

\begin{tabular}{lcccc}
\hline \hline$v_{i}(s)$ & {$[1][2][3]$} & {$[12][3]$} & {$[13][2]$} & {$[23][1]$} \\
\hline 1 & 1 & 2 & 2 & -2 \\
2 & 1 & 2 & -2 & 2 \\
3 & 1 & -2 & 2 & 2 \\
\hline
\end{tabular}

We assume that all players have an equal chance of being the proposer in every state, and players are very patient. In this example, the grand coalition [123] is not considered because it yields arbitrarily low payoffs to every player, say.

For every $\delta$ sufficiently close to one, this economy has a unique equilibrium. The limit as $\delta$ goes to one of this equilibrium is such that equilibrium payoffs and equilibrium transition probabilities, for each state, converge to the values indicated in figure 2 . The efficient state 
[12][3]

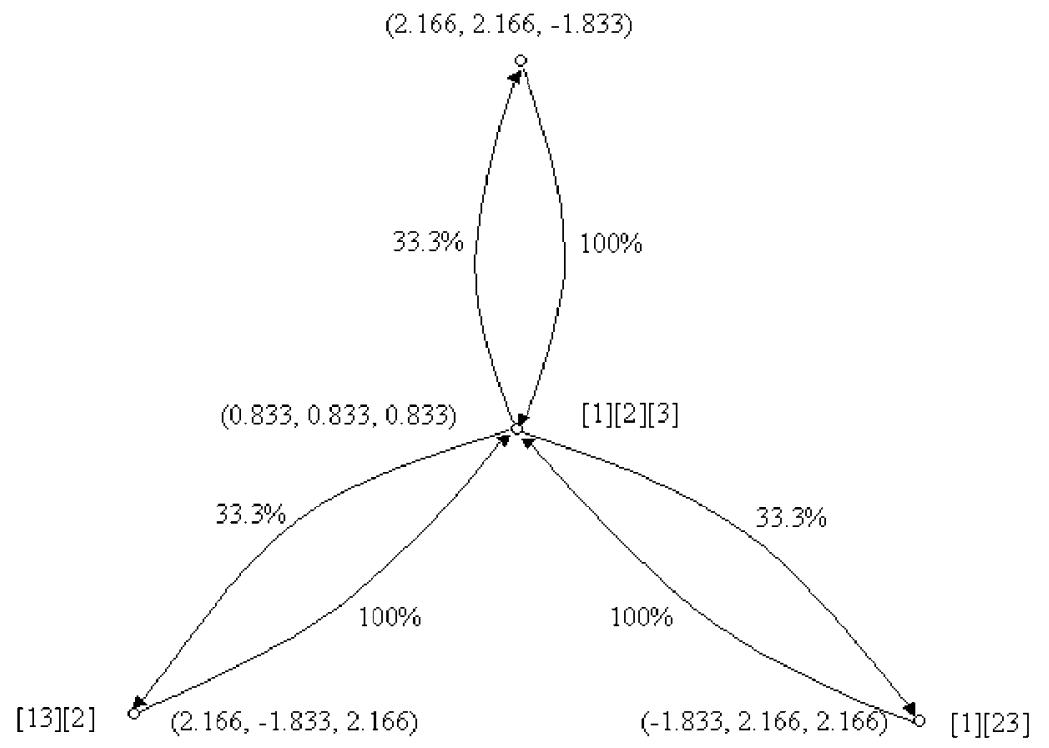

Fig. 2.-Coalitional bargaining game equilibrium values (in parentheses) and transition probabilities (percentages).

of this economy is [1][2][3]. But the system does not converge to [1][2][3], since all states are visited infinitely often in the long run.

Why doesn't the system stay in state [1][2] [3]? If that were the case, each player would get a payoff of 1 . But, player 1 , say, could achieve a strictly higher payoff by proposing to player 2 to form a coalition (i.e., move to [12][3]). Hence, the system cannot stay in [1][2][3], and it must cycle. It turns out that the only equilibrium cycle is the one shown above in which all states may occur in equilibrium.

The same example can also be used to show a role for intermediaries in coalition formation. Specifically, consider a fourth, dummy, playerreferred to as player 4 -in the above example. He is considered as dummy because he never considers joining any existing coalition (he is always in a solo coalition) and he gets the same payoff no matter how other players are partitioned into coalitions. Also, we assume that all four players are proposers with equal probability in all states and the discount rate is infinitesimally close to one. We can show that there exists an equilibrium in which player 4 makes positive profits even though all players are very patient and player 4's consent is not required for any transition. Such a result suggests a positive role for intermediaries in our setup.

At first sight, it might seem counterintuitive that a player who has no 
intrinsic role in the economy makes positive profits in the limit. Indeed, if players 1,2 , and 3 desire collectively to move from state $s$ to state $s^{\prime}$, why can't they do it themselves leaving no rent to player 4 ?

The problem is that there is no consensus among players 1,2 , and 3 about which pattern to follow. In the disaggregate state [1] [2][3] [4], player 1 is eager to form a coalition with either player 2 or 3 , but he is very afraid that players 2 and 3 might form a coalition themselves (if they happen to have the lead in making the proposal at that state). This in turn implies that, when player 4 is the proposer at state [1] [2] [3] [4], player 1 is ready to pay player 4 some significant amount so that the system moves to state [12][3][4], say, and the risk that players 2 and 3 move to [1][23][4] is avoided (in fact is reduced and postponed).

\section{Exchange Economies}

A third major application of our setup is exchange economies. Consider an economy with $n$ agents, $N=\{1, \ldots, n\}$, and $m$ indivisible goods. Agents start with some endowment, and they can exchange their commodities over the various time periods. Note that our setup does not allow for long-term contracts; hence we do not allow for exchanges of financial assets such as futures or options ${ }^{26}$ In each period, the state space $Z$ of the economy is represented by the profile $\omega$ of allocations $\left(\omega_{i}\right)_{i=1}^{n}$, where $\omega_{i}$ is agent $i$ 's allocation in the current period and $\omega_{i} \cap$ $\omega_{j}=\varnothing$ for all $i, j \in N$ (joint ownership is not allowed).

As the notion of property right suggests, a move from state $\omega$ to state $\omega^{\prime}$ requires the consent of agent $i$ whenever agent $i$ 's allocation is modified, that is, $\omega_{i} \neq \omega_{i}^{\prime}$. Thus the transition rule for the exchange economy is that for any $\omega, \omega^{\prime}$ and subset $S$ of agents, $\omega \rightarrow_{S} \omega^{\prime}$ if $\left\{i \in N: \omega_{i} \neq\right.$ $\left.\omega_{i}^{\prime}\right\} \subseteq S$. Clearly, such a transition rule satisfies assumptions $1-4 .{ }^{27}$

We do not make any restriction as to the probabilities $p_{i}(\omega)$ that agent $i$ makes the offer in state $\omega$. Thus even someone whose allocation does not change may propose a trade from $\omega$ to $\omega^{\prime}$. This, in particular, allows us to analyze the role of intermediaries in exchange economies.

We further assume that agents have the same discount factor $\delta=$ $1 /(1+r)$, where $r$ can be interpreted as the interest rate in the economy.

Regarding the payoff specification, we distinguish between exchange economies with and without externalities: $(a)$ In the no-externality case, the per period payoff of player $i$ in state $\omega$ is $(1-\delta) u_{i}\left(\omega_{i}\right)$, where $u_{i}$ stands for agent $i$ s utility function. (b) In the externality case, the per period payoff of player $i$ for a given allocation profile $\omega$ does not solely

\footnotetext{
${ }^{26}$ Our model is better adapted to economies with less developed financial markets.

${ }^{27}$ In production economies in which investments can be made prior to the transactions, assumption 4 would be violated. The general analysis of collusion-proof spot contracts when assumption 4 is not met should be the subject of further research.
} 
depend on $\omega_{i}$ but on the entire allocation profile; that is, it has the form $(1-\delta) u_{i}(\omega)$.

Related literature.-(1) Bargaining setups of exchange economies without externalities were first studied by Rubinstein and Wolinsky (1985) and Gale (1986). Note that our setup allows for multilateral exchanges, whereas Rubinstein-Wolinsky and Gale focus on bilateral exchanges. Note also that Gale and Rubinstein-Wolinsky assume that there is only one time of consumption, whereas our setup uses a flow formulation for payoffs. Our setup is thus more adapted to deal with exchange economies of durable goods. ${ }^{28}$ (2) Bargaining setups of exchange economies with externalities were first studied by Jehiel and Moldovanu $(1995 a, 1995 b, 1999)$ in the one-object case. Our setup allows for an arbitrary number of goods.

Our analysis delivers several insights into the exchange economy application. First, when there are no externalities, the economy must result in an efficient allocation of goods in a finite number of steps. This result is reminiscent of and complements the insights of Gale (1986), which were obtained in a slightly different setup with infinite populations, no discounting, and nondurable goods (see above). Efficiency obtains here because the efficient state is negative externality-free in this case.

In exchange economies with externalities, several interesting dynamics may emerge. Sometimes cycles between states may emerge in equilibrium or, as the following example illustrates, the economy may converge to an inefficient state. To illustrate this possibility, consider an exchange economy with one asset $A$ and three agents. The agents' utilities are contingent on the identity of the owner of the asset, as shown below:

\begin{tabular}{lccc}
\hline \hline$v_{i}(s)$ & $\omega_{1}=A$ & $\omega_{2}=A$ & $\omega_{3}=A$ \\
\hline 1 & 7 & 0 & 0 \\
2 & 0 & 6 & -3 \\
3 & 0 & 0 & 7 \\
\hline
\end{tabular}

All proposers are chosen with equal probability in all allocation configurations. In figure 3 we show the unique equilibrium payoff and transition probabilities for $\delta$ infinitesimally close to one.

Even though the state in which player 2 owns the asset is inefficientit yields a welfare of 6 whereas player 1 owning the asset yields a welfare of 7-it is stable. Player 2 is not willing to sell the asset to player 1 because she knows that whenever 1 gets the asset, instead of keeping it, he will sell it at a profit to player 3 . There are profits to be made

\footnotetext{
${ }^{28}$ In Gale's (or Rubinstein and Wolinsky's) setup the offer is made by either of the parties who exchange their goods, whereas in our model intermediaries may propose trades to whomever they wish.
} 


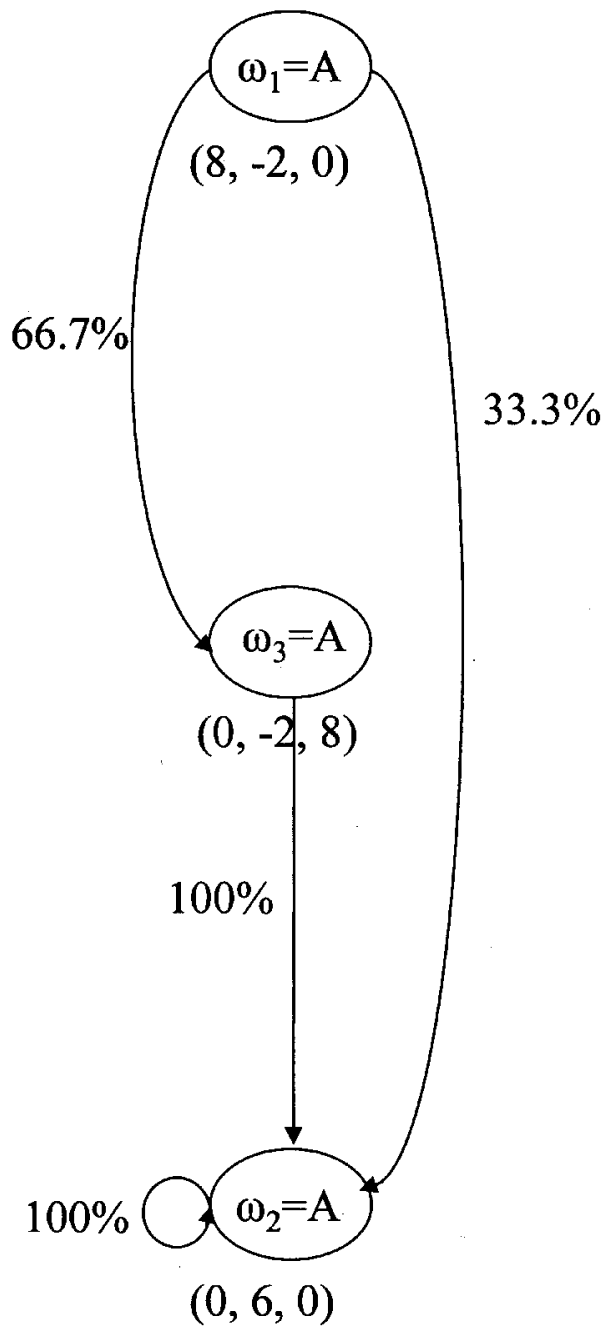

FIG. 3.-Exchange economy equilibrium values (in parentheses) and transition probabilities (percentages).

with this inefficient transaction at the expense of player 2, because when player 3 owns the asset, he imposes a significant negative externality on player 2.

To conclude this application section, observe that the various examples presented above all contained inefficiencies or rich dynamics (i.e., cycles). The fundamental reason for inefficiencies or rich dynamics is the fact that the efficient state, say $z^{\text {eff }}$, is not negative externalityfree. That is, it is possible to leave state $z^{\text {eff }}$ without the consent of player 
$j$ for, say, state $z^{\text {ineff }}$, and player $j$ is worse off (in terms of flows of payoffs) in state $z^{\text {ineff }}$ than in state $z^{\text {eff }}$. Our general analysis thus permits a fine understanding of the causes of inefficiencies in dynamic processes of social and economic interactions in which only collusion-proof spot contracts can be proposed.

\section{Contracts and Collusion}

The purpose of this section is to provide some preliminary results such as the existence and characterization of the equilibrium of our main model as well as to provide a contract-theoretical motivation for our model. In our model, offers take a simple form: take-it-or-leave-it offers in which any approached agent can block its implementation. But we shall show that such a restriction on offers emerges endogenously in situations in which agents are unable to commit to actions to be taken in future stages but are otherwise unconstrained both in terms of the spot contracts they can offer and in terms of collusive mechanisms that the approached agents can arrange in response to the proposed spot contract (a collusion-proof spot contract setup).

A reader willing to see the analysis of our simple take-it-or-leave-it offer game may read Subsection $A$ and then jump to Section $V$ (thereby skipping the contract-theoretic discussion made in Subsecs. $B-D)$.

\section{A. Basic Properties of the Simple-Offer Economy}

Let $\sigma$ be an MPE of economy $\mathcal{E}(\rightarrow, v, p, \delta)$. For every state $a$ and player $i$, we let $\phi_{i}(a)$ represent the associated expected equilibrium value of player $i$ when the system is in state $a$ (for simplicity, we omit the dependency on $\sigma$ ). If the system moves to state $a$, player $i$ 's continuation value (gross of transfers) is given by $x_{i}(a)$, where

$$
x_{i}(a)=\delta \phi_{i}(a)+(1-\delta) v_{i}(a) .
$$

That is, player $i$ receives the flow of payoff $(1-\delta) v_{i}(a)$ for the current period, and at the start of next period the system is in state $a$, resulting in a payoff of $\delta \phi_{i}(a)$ for player $i$.

In the following analysis it will be convenient for any Markovian strategy $\sigma_{i}$ of player $i$ to denote by $\sigma_{i}[a](S, b, t)$ the associated probability that offer $(S, b, t)$ is made by player $i$ at state $a$ when $i$ is the proposer at that state. It will also be convenient to let $\sigma_{i}(a)(S, b)$ denote the associated probability that player $i$ at state $a$ makes a proposal to coalition $S$ to move to state $b$, and to let supp $\sigma_{i}(a)$ denote the support of $\sigma_{i}(a)$.

Whenever the strategy $\sigma_{i}(a)$ satisfies $\sigma_{i}(a)(S, b)>0$, that is, with a positive probability, in state $a$ player $i$ makes a proposal to players in $S$ to move to state $b$ such that $a \rightarrow_{s} b$, the (equilibrium) transfer that player 
$i$ proposes to $j$ is $t_{j}=x_{j}(a)-x_{j}(b)$. This is indeed the minimum transfer required by $j$ to accept the transition from $a$ to $b$, and it is such that player $j$ is indifferent between rejecting and accepting the offer (since $\left.t_{j}+x_{j}(b)=x_{j}(a)\right)$. Thus, when player $i$ at state $a$ approaches $S$ to move to state $b$, transfers are uniquely determined, and the equilibrium strategy of player $i$ can be fully inferred from $\sigma_{i}(a)$. The above argument also shows that in equilibrium, player $i$ at state $a$ will approach coalition $S$ and propose a transition to state $b$, where $(S, b)$ maximizes $\sum_{j \in S}\left[x_{j}(b)-x_{j}(a)\right]$ over all feasible transitions, that is, such that $a \rightarrow_{S} b$.

We establish next that an MPE always exists and is characterized by the following properties (see the Appendix for the proof and a discussion of other basic properties).

Proposition 1. There exists at least one MPE for all economies $\mathcal{E}(\rightarrow, v, p, \delta)$. Moreover, a strategy profile $\sigma$ is an MPE whenever the following conditions hold: (i) The support of $\sigma_{i}(a)$ satisfies

$$
\operatorname{supp}\left[\sigma_{i}(a)\right] \subset \arg \max _{(S, b)}\left\{\sum_{j \in S}\left[x_{j}(b)-x_{j}(a)\right]: a \rightarrow_{S} b \text { and } i \in S\right\} .
$$

(ii) For all $i$ and $a$,

$$
\phi_{i}(a)=\sum_{j \in N} p_{j}(a) \phi_{i}^{j}(a)
$$

where $^{29}$

$$
\phi_{i}^{j}(a)= \begin{cases}x_{i}(a)+\max _{(b, S)}\left\{\sum_{j \in S}\left[x_{j}(b)-x_{j}(a)\right]: a \rightarrow_{S} b \text { and } i \in S\right\} & \text { if } j=i \\ \sum_{(S, b)} \sigma_{j}(a)(S, b)\left[I(i \in S) x_{i}(a)+I(i \notin S) x_{i}(b)\right] & \text { if } j \neq i\end{cases}
$$

is player $i$ 's equilibrium payoff at state $a$ when player $j$ is the proposer at that state.

\section{B. Long-Term Contracts}

Suppose in contrast to our main simple-offer contracting scenario that agents are free to propose and sign long-term contracts; that is, contracts involving actions or nonactions to be taken in future stages are allowed.

In such a case, efficiency must obtain at the start of the interaction when there is no contractual agreement yet. The reason is that if some inefficiency were to occur when there is no contract yet, it could easily

${ }^{29}$ The term $I$ is the indicator operator. Accordingly, $I(E)=1(0)$ if $E$ is true (false). 
be overcome by proposing a contract specifying that the economy will stay indefinitely in the efficient state and also specifying appropriate lump-sum payments so that each agent gets more than what he would have gotten otherwise if the contractual offer were rejected. If such a contract is proposed with the clause that if someone objects to it the contract is withdrawn, it must be accepted in any MPE of the underlying unlimited contracting game.$^{30}$ Hence, efficiency obtains immediately in all MPE of the corresponding game. This result can be viewed as an illustration of the celebrated Coase theorem.

\section{General Spot Contracts}

Suppose now that agents are not able to use long-term contracts but are able to use general spot contracts in which allocations and transfers are contingent on players' responses. That is, the restriction we impose on contracts is that they cannot stipulate actions or nonactions to be made at future stages; but in contrast to the simple-offer contracting scenario, one need not stay in the current state if one or several approached agents refuse the contract. That is, even if a subset of the approached agents $S$ refuses the contract, a move from the current state $a$ to another state $z(A)$ with side payments between agents in $A$ can still be implemented provided that all agents in $A \subset S$ have accepted the contract; the transition is feasible with the sole permission of agents in $A$, that is, $a \rightarrow A_{A} z(A)$, and the contract had stipulated this in advance. We have the following proposition.

Proposition 2. All MPE of the economy with general spot contracts are efficient, entailing an immediate move to the efficient state, where it remains forever.

The MPE of the economy with general spot contracts can be analyzed using the basic insights of static economies with general commitments (Jehiel et al. 1996; Segal 1999): The proposer should include everyone in the contract, the outcome of the contract should be efficient, and the proposer $i$ should extract payment from all other agents $j, j \neq i$, by threatening each agent $j$ to implement the worst outcome for $j$ if agent $j$ refuses the contract (see in particular Jehiel et al. [1996]).

In our dynamic setting, the same logic applies, with the significant difference that continuation values are endogenously determined. Roughly, the insight from the static literature ensures that whatever the current state and the identity of the proposer, there should be an immediate move to the same state $a^{*}$ (i.e., the state that maximizes

\footnotetext{
${ }^{30}$ The Markovian assumption is used to ensure that if the contract is not approved, the ensuing payoff does not depend on the reasons that caused the contract not to be approved.
} 
$x_{N}(a)=\sum_{i=1}^{n} x_{i}(a)$, where $x_{i}(a)$ is the continuation value of player $i$ whenever the system moves to state $a$ ). From this property of the equilibrium transition, we are able to infer (see the Appendix) that this state $a^{*}$ is necessarily the efficient state, that is, $a^{*} \in \arg \max _{a \in Z} \sum_{i=1}^{n} v_{i}(a)$.

It is somewhat surprising that long-term contracts are not really needed in order to achieve an efficient outcome. The efficiency result is slightly surprising in light of the fact that players are not strategically moving to states in which their ability to impose negative externalities on other players in the future is increased. Rather, just the use of static threats is enough to sustain efficient outcomes in which there is an immediate move to the Pareto-efficient state.

To illustrate the equilibrium in which general spot contracts are allowed, reconsider the legislative bargaining example of Section III.A, allowing now for general spot contracts. For any discount rate, the equilibrium values are just $\phi_{i}(s)=1.6$ for all states $s=a, b, c$, and $d$. The equilibrium strategy at any state is for proposers to make an offer to the set $N$ of all agents to move to state $d$ with a threat to any player rejecting the offer to move to the state that is most undesirable to him (e.g., if 1,2 , or 3 rejects the offer made by whoever is the proposer, then the state moves to $c, b$, or $a$, respectively). By threatening to move to the state that is most undesirable to responders if they reject the offer, the proposer is able to extract all the short-term surplus of $4.8(1-\delta)$ for himself, and so his value is equal to $4.8(1-\delta)+1.6 \delta$ and every responder's value is equal to $1.6 \delta$. Taking into account that all players have the same probability of being the proposer yields that the values should be equal to 1.6 in all states.

\section{Collusion-Proof Spot Contracts}

Consider now a situation in which agents are allowed to make any spot contract offer to any group of their choice as defined in the previous subsection, but assume now in addition that the agents approached by the proposer are allowed to react to the main contract by forming a collusive ring and by coordinating their responses (we also allow for side payments between ring members). Specifically, we model the collusive stage by assuming that if agent $i$ offers a spot contract to the subset of agents $S \backslash\{i\}$, then the agents receiving the offer can hire a third party whose objective is to maximize the sum of their payoffs. The third party responds to proposer $i$ s contract offer in place of agents $j \in S \backslash\{i\}$, and she can also implement transfers among the ring members $S \backslash\{i\} .{ }^{31}$ This modeling of collusion agrees with how Laffont and Martimort (1997)

\footnotetext{
${ }^{31}$ How the third party shares the surplus (if any) between the ring members is left unspecified. However, for the analysis that follows, how this surplus is shared is irrelevant.
} 
model collusion in mechanism design (see also Graham and Marshall 1987; McAfee and McMillan 1992; Caillaud and Jehiel 1998). Somehow allowing both for general spot contracts and for collusion is a way to allow for the same commitment abilities both on the side of the proposers and on the side of the approached agents.

Our main result is that the equilibria in both the simple-offer contract (considered in Sec. II) and the collusion-proof spot contract models are equivalent under assumption 4 that no solo player can change the state on his own. They are equivalent in the sense that given any MPE of one model, there is an MPE of the other model with the same equilibrium values for all players at all states and the same transition probabilities from state to state induced by the equilibrium strategy profile. ${ }^{32}$

Proposition 3. The MPE of the collusion-proof spot contract and the simple-offer model are equivalent, whenever the no-solo move player assumption 4 holds.

Thus proposition 3 implies that the proposer's benefit due to his ability to use general spot contracts is neutralized by responders' ability to coordinate their response.

The result holds under the assumption that a proposer alone cannot move from the current state. ${ }^{33}$ Under this assumption, the possibility of collusion means that the set of approached agents, by all coordinating to refuse the contract, can always secure a payoff equal to the continuation value that they would have gotten if the system had stayed at the current state. ${ }^{34}$ The proposer is thus forced to make an offer that gives responders at least this minimum amount (otherwise collusion will take care of it). It is not difficult to see (see the Appendix) that the simpleoffer contracts allow the proposer to reduce the rent of the approached agents to the minimum required amount while avoiding any further possibility of collusion. Therefore, the proposer finds it optimal to use simple-offer contracts, and the equivalence of the two contracting scenarios follows.

\section{Convergence, Stability, and Efficiency Analysis}

In this section we explore the dynamics and efficiency properties of situations in which only simple-offer contracts can be proposed or, equiv-

\footnotetext{
${ }^{32}$ Note that the strategy spaces of both models are different (the extensive forms are also slightly different), and thus it does not make sense to directly compare the strategy profiles of both models.

${ }^{33}$ If a solo player can change the state on his own, the above conclusion is not true since a player may now possibly use the threat to move to a less favorable state as one or more agents refuse the deal. Yet in most applications of interest (as we noted above), a solo player cannot change the state, and assumption 4 holds.

${ }^{34}$ In the legislative bargaining example considered in Secs. III. $A$ and IV. $C$, this collusion possibility prevents, say, agent 1 in the efficient state $d$ from extracting payments from 2 and 3 just to stay in state $d$ (in order to avoid moving, respectively, to state $b$ or $a$ ).
} 
alently under assumption 4 (see Sec. IV), in which only collusion-proof spot contracts can be proposed. We have already provided a number of applications with rich dynamics (see Sec. III), and we wish now to analyze further the reasons for these dynamics obtained in highly specific examples.

We first analyze the asymptotic behavior of the process and its sensitivity to the initial state. Our main result is that the long-run behavior of the process is independent of the specification of the initial state. We then study the efficiency and stability properties of the equilibria. Our main result is that a robust condition that guarantees the stability and efficiency of the economy is the existence of a state that is efficient and such that no move away from it imposes negative externalities on excluded players-an ENF state.

\section{A. Long-Run Behavior}

Any equilibrium $\sigma$ of the economy $\mathcal{E}(\rightarrow, v, p, \delta)$ induces a transition probability in the state space $\mu(a, b)=\sum_{j \in N} \sum_{S} p_{j}(a) \sigma_{j}(a)(S, b)$, where $\mu(a$, $b$ ) denotes the equilibrium probability that the system once in state $a$ moves to state $b$.

The equilibrium long-run behavior of the economy is described by the stable sets (or ergodic classes) induced by $\mu$. The stable sets of the economy are defined by two properties: (i) starting from any state that belongs to a stable set, the process remains in the stable set forever, and (ii) no (nontrivial) subset of a stable set is stable. ${ }^{35}$ By a well-known result of the theory of Markov chains, starting from any state, the process converges, in a finite number of steps, to a stable set (see Doob 1953). Therefore, the stable sets describe the long-run behavior of the economy.

When a stable set has a unique state, we refer to it as a stable state or absorbing state. Note that when a stable set contains several states, the system cycles between these states. States that do not belong to a stable set are also referred to as transient states.

The first proposition of this section establishes that the aggregate welfare must be approximately the same in all states as players get very patient.

Proposition 4. The aggregate equilibrium values are approxi-

\footnotetext{
${ }^{35}$ The stable sets are the sets $E \subset Z$ such that (i) (closedness) for any $a \in E$, there exists no $b \in Z \backslash E$ such that $\mu(a, b)>0$; and (ii) (irreducibility) for any $a, b \in E$, there exists a sequence $a=a_{0}, \ldots, a_{k}, \ldots, a_{m}=b$ with $a_{k} \in E$ and $\mu\left(a_{k-1}, a_{k}\right)>0$.
} 
mately the same at all states for all economies $\mathcal{E}(\rightarrow, v, p, \delta)$ if players are patient enough ( $\delta$ close to one) ${ }^{36}$

Certainly, the aggregate levels of efficiency are the same at all states in the same ergodic class. The level of efficiency of an ergodic set is given by the weighted average of the aggregate payoffs of the states in the ergodic set, where the weights are given by the frequency of time the economy spends in each state (the invariant probability measure). However, a priori, it could be that two different ergodic sets may have different levels of efficiency. This possibility, though, is ruled out by proposition 4. Intuitively, if there were two ergodic sets with two different aggregate welfare levels, there would be at least one player who would be able to benefit from a move from the less efficient ergodic set to the more efficient ergodic set (see lemma 1 in the Appendix). Thus this player would link the two ergodic sets by his move, which means that the two ergodic sets would have to be in fact a single ergodic set (a contradiction). The rest of the argument consists in observing that when players are very patient, the aggregate welfare at any transient state depends only on the aggregate welfare at ergodic sets because the system moves in a finite number of steps to an ergodic set, and so the utility flows at transient states are negligible (irrelevant in the limit of arbitrarily patient players).

Our next result shows that while multiple ergodic classes are possible, generically, ${ }^{37}$ any equilibrium has only one ergodic class whenever players are sufficiently patient. Thus the long-run behavior of the dynamic process (not only the aggregate welfare) is the same regardless of the initial state from which the system starts. And this conclusion holds true whether the system converges to a single state or cycles between several states.

Proposition 5. The long-run properties of economies $\mathcal{E}(\rightarrow, v, p$, $\delta)$ are not dependent on the initial state if players are patient enough. Formally, for generic economies, there exists $\bar{\delta}<1$ such that, if $\delta \geq \bar{\delta}$, all equilibria have only one stable set.

The intuition for proposition 5 is roughly as follows. Suppose that there is a sequence (as $\delta$ tends to one) of equilibria of the economy with discount factor $\delta$ such that every equilibrium has two ergodic sets $E$ and $E^{\prime}$. The equilibrium transitions governing the states in $E$ (respectively $E^{\prime}$ ) solely depend on the payoff (and transition structure) in states in $E\left(E^{\prime}\right)$. And so do the limits of these equilibrium transitions as

\footnotetext{
${ }^{36}$ Formally, the result means that $\lim \max \left\{\left|\phi_{N}^{(\delta)}(a)-\phi_{N}^{(\delta)}(b)\right|: a, b \in Z\right\}=0$,

where $\phi_{N}^{(\delta)}(\cdot)=\sum_{i \in N} \phi_{i}^{(\delta)}(\cdot)$ is the aggregate equilibrium payoffs associated with any equilibrium $\sigma^{(\delta)}$ of the economy $\mathcal{E}(\rightarrow, v, p, \delta)$.

${ }^{37}$ Except in a subset of the set of payoffs $v \in R^{n \cdot|Z|}$ of Lebesque measure zero.
} 
$\delta$ goes to one. But, by proposition 4 , the limit of the aggregate value in ergodic set $E$ must be the same as in ergodic set $E^{\prime}$. This cannot hold generically because if, say, one modifies only the payoffs in states in $E$, the aggregate welfare will not change in $E^{\prime}$ whereas it will in $E$, violating the equal welfare property. Thus proposition 5 must hold.

Proposition 5 has important implications, since it shows that there is no point (for long-run considerations) in reinitializing the process to another state if one is to leave the structure of transitions unchanged. For example, in exchange economies with transferable utilities (with or without externalities), this result implies that the initial allocation of property rights plays no role in the long-run allocation of goods. And so policies that would consist in reallocating the goods initially would have no effect on the long-run properties of the economy. ${ }^{38}$

Remark.-It should be noted that proposition 5 (and also proposition 4) assumes that players are sufficiently patient. When players are not patient enough, there may be equilibria with multiple stable sets (with different associated aggregate welfare) ${ }^{39}$

\section{B. Efficient Negative Externality-Free States}

What are the efficiency and stability properties of equilibria? We shall show that the answer depends on whether or not there exists an ENF state, a key concept introduced in the paper. In short, an efficient state is an ENF state if any group of agents that can move the economy to another state (possibly in several steps) cannot in the process impose negative externalities on players that have been excluded from the move. When we require robustness with respect to the (possibly state-dependent) probabilities that the various players are selected to make proposals, the existence of an ENF state is a necessary and sufficient condition for efficiency and stability.

The precise definition of an ENF state follows.

Definition 3. (i) A state $a$ of the $v$ economy $\mathcal{E}(\rightarrow, v)$ is negative externality-free if and only if, for all players $i \in N$ and moves $a \rightarrow_{S_{1}}$ $a_{1} \rightarrow \cdots \rightarrow_{S_{n}} a_{n}=b$ by subcoalitions $S_{k} \subset N \backslash i$ (excluding player $i$ ),

\footnotetext{
${ }^{38}$ Such a result is reminiscent of Coase (1960) and can be viewed as a significant extension of Jehiel and Moldovanu (1999) to the multiobject case (with externalities) and infinite-horizon bargaining contexts.

${ }^{39}$ For example, consider a voting problem in which three legislators have the following utility with respect to four policy choices (players have equal probabilities of being proposers): $v(a)=(1.5,1,0), v(b)=(1,0,1.5), v(c)=(0,1.5,1)$, and $v(d)=(1,1,1)$. Any of the policies $a, b$, or $c$ can be approved and changed by a simple majority (at least two legislators), and policy $d$ requires unanimity to be approved and changed. In this example there are two stable sets for all discount rates $\delta<0.75:\{a, b, c\}$ and $\{d\}$.
} 
$v_{i}(b) \geq v_{i}(a)$. (ii) A state is an ENF state if and only if it is efficient and is negative externality-free. ${ }^{40}$

So, according to our definition, a state $a$ is free of negative externalities if no group $S$ that can implement a move from $a$ to $b$ (possibly in several steps) can reduce the flow of payoffs of players outside $S$ (i.e., $v_{i}(b) \geq v_{i}(a)$ for all $\left.i \notin S\right)$. ENF states are those states that are both efficient and free of negative externalities. ${ }^{41}$ Observe that the requirement of unanimous consent to move away from one efficient state implies the existence of an ENF state. So does the absence of (negative) externalities in coalition formation bargaining games or in exchange economies (see the application section).

Our first result of this section establishes that ENF states are stable regardless of the patience of the players and, in addition, that economies with an ENF state are asymptotically efficient in the sense that, for very patient players, the system stabilizes to states that are efficient in the long run (after a number of possible transitory moves). ${ }^{42}$ Note that asymptotic efficiency should be contrasted with strong efficiency, which requires that, starting from any given state, there is an immediate move to an efficient state. ${ }^{43}$ But whenever players are very patient, the potential loss induced by asymptotic efficiency relative to strong efficiency is very small (it vanishes to zero as players get infinitely patient). So asymptotic efficiency is the economically relevant measure of efficiency when one considers arbitrarily patient players.

Proposition 6. (i) Stability: All ENF states of $\mathcal{E}(\rightarrow, v)$ are stable states of any equilibrium of $\mathcal{E}(\rightarrow, v, \delta, p)$, for all $p$ and $\delta$. (ii) Efficiency: Moreover, if there exists at least one ENF state of $\mathcal{E}(\rightarrow, v)$ (i.e., $\mathrm{ENF} \neq \varnothing)$, then all economies $\mathcal{E}(\rightarrow, v, p)$ are asymptotically efficient.

Note first that since a stable state is one in which the economy stays forever, then it must be the case that $\phi_{i}\left(a^{*}\right)=v_{i}\left(a^{*}\right)$ for all $i \in N$ whenever $a^{*}$ is stable. Moreover, a necessary condition for stability of state $a^{*}$ is that the inequalities

$$
v_{S}\left(a^{*}\right) \geq x_{S}(b)=\delta \phi_{S}(b)+(1-\delta) v_{S}(b)
$$

\footnotetext{
${ }^{40}$ It is worth pointing out that we cannot weaken the definition of ENF to consider only states that are reachable in a one-step deviation instead of multiple steps, if we wish to guarantee stability (i.e., an efficient state $a$ such that, for all $a \rightarrow_{s} b, v_{i}(b) \geq v_{i}(a)$ for all $i \notin S$ may not be stable). The following example illustrates this point: $N=\{1,2,3\}$, $Z=\{a, b, c\}, v(a)=(0,0,3), v(b)=(-1,-1,4), v(c)=(1,1,0), a \rightarrow_{12} b, b \rightarrow_{12} c$, and $c \rightarrow_{123} a$. The unique efficient state is not stable, and the system cycles over all states.

${ }^{41}$ If one adds a transitivity axiom (i.e., for any three states $b, c$, and $d$ and coalition $S$, $b \rightarrow_{S} c$ and $c \rightarrow_{S} d$ imply that $b \rightarrow_{S} d$ ), then the definition can be simplified to consider only direct transitions from $a$ to $b$.

${ }^{42}$ Formally, an economy $\mathcal{E}(\rightarrow, v, p)$ is asymptotically efficient if and only if $\lim _{\delta \rightarrow 1} \phi_{N}^{(\delta)}(a)=\max _{b \in Z} v_{N}(b)$ for any equilibrium $\sigma^{(\delta)}$ of the economy $\mathcal{E}(\rightarrow, v, p, \delta)$ and any state $a \in Z$

${ }^{43}$ Strong efficiency has been shown to obtain whenever long-term contracts are available or general spot contracts are available while the approached agents cannot collude.
} 
hold for all feasible moves $a^{*} \rightarrow_{S} b$ (for all $S \subset N$ and states $b \in Z$ ), where $h_{S}(a)=\sum_{i \in S} h_{i}(a)$ for $h=v, \phi$, and $x$. These conditions ensure that no deviation away from the stable state is profitable.

The stability of an ENF state $a$ arises because any move away from state $a$, say $a \rightarrow_{s} b$, is not profitable for coalition $S$ (i.e., the excess $\left.x_{S}(b)-x_{S}(a) \leq 0\right)$ : given that $a$ is negative externality-free, coalition $S$ cannot impose any negative externality on players in $N \backslash S$, once the process is at state $a$; and given that $a$ is efficient, coalition $S$ cannot make any improvements, since all players in $N \backslash S$ can only benefit from any move that coalition $S$ makes, and $a$ is already an efficient state.

The existence of an ENF state is also a sufficient condition for asymptotic efficiency. The reason is that, according to proposition 4, the aggregate equilibrium value must be the same at all states. But if there exists an ENF state-which by condition i of proposition 6 must be stable-then in any equilibrium the aggregate welfare at the ENF state is the Pareto-efficient level. Therefore, the aggregate value at any other state must also be asymptotically close to the Pareto-efficient level.

Naturally, since economies without negative externalities or economies such that the efficient state can be left only with unanimous consent have ENF states, such economies must converge in a finite number of steps to the efficient state and remain there thereafter. Proposition 6 has several practical implications. For example, it can be used to establish asymptotic efficiency in exchange economies without externalities (see Sec. III.C), which is reminiscent of Gale (1986), and in coalitional bargaining games with characteristic function forms (see Sec. III.B). In addition, it can be used to establish asymptotic efficiency in coalitional bargaining games with externalities in which the grand coalition is efficient (see Gomes, forthcoming) or legislative bargaining in which unanimity is required to change the efficient policy.

Proposition 6 has established that the existence of an ENF state is sufficient to guarantee the convergence to an efficient state when players are sufficiently patient. But, is the existence of an ENF state necessary? When we require robustness with respect to the (possibly state-dependent) probabilities that the various players are selected to make proposals, the existence of an ENF state is also a necessary condition for stability and asymptotic efficiency. More precisely, we have the following proposition.

Proposition 7. (i) Stability: Any state of $\mathcal{E}(\rightarrow, v)$ that is not negative externality-free is not a stable state of $\mathcal{E}(\rightarrow, v, \delta, p)$ for $\delta$ close to one and for some proposers' probabilities $p$. (ii) Efficiency: Moreover, any economy $\mathcal{E}(\rightarrow, v)$ without any ENF states is such that there exist (an open set of) proposers' probabilities $p$ such that $\mathcal{E}\left(\rightarrow, v^{\prime}, \delta, p\right)$ is not asymptotically efficient, for almost all $v^{\prime}$ in a neighborhood of $v$.

If there is no negative externality-free state, then from any state $a$ 
there is a move by coalition $S$ (possibly in several steps) to another state $b$ such that player $i \notin S$ s flow of payoff is lower at state $b$ than at state $a$. Condition i of proposition 7 shows that in such a situation any given state $a$ is not stable for some specifications of $p$. Intuitively, instability of $a$ will occur when the probability that player $i$ is the proposer at state $b$ is sufficiently small. Under such circumstances, state $a$ will not be stable because coalition $S$ would rather leave state $a$ to move to state $b$ in order to exploit the weak bargaining position of player $i$ at state $b$. In a similar vein, condition ii of proposition 7 shows that the existence of an ENF state is required for asymptotic efficiency to obtain (irrespective of proposers' probabilities). The reason is that if the efficient state is not negative externality-free, condition i of proposition 7 shows that it can be destabilized for some specifications of proposers' probabilities. Hence, asymptotic efficiency cannot obtain for such proposers' probabilities. ${ }^{44}$

It is important to observe that proposition 7 does not rule out the possibility that the economy converges to an inefficient state or to an efficient state that is not negative externality-free..$^{45}$ But, when there is no ENF state and players are patient enough, inefficiencies and cycles must occur at least for some specifications of the proposers' probabilities.

In summary, inefficiencies and instabilities are driven by negative externalities that can be imposed on players from the efficient state and moves to (inefficient) states that may enhance a player's bargaining position at the cost of inducing inefficiencies or distortions in the allocation of resources. However, as we have demonstrated in proposition 6 , the bargaining power of players creates no distortions if there exists at least one ENF state.

Our analysis mostly focused on the case of very patient players. When players are myopic, a key concept well studied in the cooperative game literature is the core defined as the set of states $a$ such that, for all coalitions $S \subset N$ and states $b \in Z$ with $a \rightarrow_{S} b, v_{S}(a) \geq v_{S}(b) .{ }^{46}$ Observe

\footnotetext{
${ }^{44}$ Observe that in proposition 7 it is important to consider perturbations of the payoff. For example, if all states of the economy are efficient, then even though there may be no ENF state, the economy is certainly (trivially) asymptotically efficient.

${ }^{45}$ See the exchange economy example developed in Sec. III. $C$ in which we get convergence to an inefficient state.

${ }^{46}$ Even though our definition of core corresponds to the classical concept, the application of the concept to coalitional bargaining games leads to some notable differences. For example, in our formulation the core of a superadditive characteristic function game is always nonempty (it is equal to the grand coalition state), whereas under the standard interpretation it is sometimes empty. The distinction comes not from the concept of core per se but from the transition relation that specifies the possible moves by coalitions. Implicit in the cooperative interpretation is the view that a subcoalition can break up from a coalition without the consent of the players left behind (see also the discussion in Sec. III.B). But this view is inconsistent with the idea that once players have agreed to form a coalition, it cannot be reorganized without the consent of all players in the coalition. We believe that in an explicit dynamic setup in which players have agreed to form the grand
} 
that the conditions for stability expressed in inequalities (5) are the same as the conditions for the core when $\delta$ is set equal to zero, that is, with myopic players. However, nonmyopic or farsighted players, when considering deviations from a state, put weight $\delta$ on the expected equilibrium value (which incorporates the expected value of all future payoffs) of the deviant coalition in the new state, as opposed to putting all the weight on the coalitional value in the new state (which is the case in the definition of the core).

In a myopic economy, there is an intimate relationship between stable states and the core (in particular, all stable states of the economy $\mathcal{E}(\rightarrow$, $v, 0, p)$ are contained in the core). Our analysis has demonstrated that in farsighted economies, stability is better captured by the concept of ENF. Thus the ENF concept plays a role for economies with very patient players similar to the role of the core for economies with very impatient or myopic players.

\section{Conclusion}

The main lessons of the paper are as follows. Whenever agents are unable to commit to actions to be made at a future stage (but are otherwise unconstrained both in their ability to propose arbitrary spot contracts and in their ability to react jointly to a proposed contract), long-run efficiency is not guaranteed. Agents constantly try to improve their bargaining position vis-à-vis other agents, and this may be a source of instability and inefficiency. But when the efficient state is negative externality-free (in the sense that if a coalition can move away from the efficient state to some alternative state without the consent of some agent, then this agent should get a flow of payoff at least as large in the new state as in the efficient state), the search for better bargaining positions is not a source of inefficiency, and convergence to the efficient state must arise if players are patient enough, in a finite number of steps. Furthermore, we also find that when there are long-run inefficiencies, changing the initial state has no effect if players are sufficiently patient. It is thus more important to design transitions guaranteeing the existence of an efficient negative externality-free state than to implement a fine initialization of the process.

coalition, it makes more sense (at least in some contexts) to assume that breaking that grand coalition apart requires the consent of all players. 
Appendix

\section{Proof of Propositions}

Proof of Proposition 1

The necessary part of the characterization follows directly from the discussion before the statement of the result and the definition of an MPE solution. The sufficient part of the characterization follows from the discussion and the use of the one-stage deviation principle for infinite-horizon games. This result states that in any infinite-horizon game with observed actions that is continuous at infinity, a strategy profile $\sigma$ is subgame perfect if and only if there is no player $i$ and strategy $\sigma_{i}^{\prime}$ that agrees with $\sigma_{i}$ except at a single stage $t$ of the game and history $h^{t}$, such that $\sigma_{i}^{\prime}$ is a better response to $\sigma_{-i}$ than $\sigma_{i}$ if history $h^{t}$ is reached (see Fudenberg and Tirole 1991).

To prove the existence, let the map $f: R^{d} \times \Sigma \rightarrow R^{d}$ be defined as

$$
\begin{aligned}
f_{i}(a)(x, \sigma)= & (1-\delta) v_{i}(a)+\delta\left(p_{i}(a) \sum_{(b, S)} \sigma_{i}(a)(S, b)\left\{\sum_{j \in S}\left[x_{j}(b)-x_{j}(a)\right]\right\}\right. \\
& \left.+\sum_{j \in N} p_{j}(a) \sum_{(b, S)} \sigma_{j}(a)(S, b)\left[I(i \in S) x_{i}(a)+I(i \notin S) x_{i}(b)\right]\right),
\end{aligned}
$$

for $(x, \sigma) \in R^{d} \times \Sigma$. Consider the set

$$
\Sigma_{i}(a)=\left\{(S, b): \text { where } a \rightarrow_{S} b \text { and } i \in S\right\},
$$

and let $\sigma_{i}(a) \in \Delta^{\Sigma_{i}(a)}$ be the set of probability distributions over $\Sigma_{i}(a)$. Let $\Sigma_{i}=$ $\times_{a \in Z} \Sigma_{i}(a)$ and $\Sigma$ be the set of offering strategies for all players.

Let the correspondence $F: R^{d} \rightarrow \rightarrow R^{d}$ be defined as

$$
F(x)=\{f(x, \sigma): \sigma \in \Sigma(x)\},
$$

where

$$
\Sigma(x)=\left\{\sigma \in \Sigma: \operatorname{supp}[\sigma(i, a)] \subset \arg \max _{(b, S)}\left\{e(a)(S, b)(x): a \rightarrow_{S} b \text { and } i \in S\right\},\right.
$$

where

$$
e(a)(S, b)(x)=\sum_{j \in S}\left[x_{j}(b)-x_{j}(a)\right]
$$

According to proposition 1, a payoff $x \in R^{d}$ is an MPE if and only if $x$ is a fixed point of $F$.

1. Let $X \subset \mathbb{R}^{m}$ be a compact and convex set in which the $a$-coordinate $x_{i}(a)$ satisfies

$$
\min _{s \in Z} v_{i}(s) \leq x_{i}(a) \leq \max _{s \in Z} v_{N}(s)-\sum_{j \in N} \min _{s \in Z} v_{j}(s)
$$

and $X=\times_{i \in N} I_{i}$. It is immediate that $F(X) \subset X$.

2. The function $F(x)$ is a convex (and nonempty) set for all $x \in X$ : Say that $z, z^{\prime} \in F(x)$ with $z=f(x, \sigma)$ and $z^{\prime}=f\left(x, \sigma^{\prime}\right)$, where $\sigma, \sigma^{\prime} \in \Sigma(x)$. Then, for any $\lambda \in[0,1]$,

$$
\lambda z+(1-\lambda) z^{\prime}=f\left(x, \lambda \sigma+(1-\lambda) \sigma^{\prime}\right) \in F(x)
$$

because $\lambda \sigma+(1-\lambda) \sigma^{\prime} \in \Sigma(x)(\Sigma(x)$ is convex $)$. 
3 . The correspondence $F$ is upper hemicontinuous; that is, for any sequence $\left(x^{n}, f\left(x^{n}, \sigma^{n}\right)\right) \rightarrow(x, z)$ with $\sigma^{n} \in \Sigma\left(x^{n}\right), z \in F(x)$ (i.e., there exists a $\sigma \in \Sigma(x)$ such that $f(x, \sigma)=z)$. The sequence $\left(\sigma^{n}\right)$ belongs to $\Sigma$, a compact subset of a finitedimension Euclidean space. Therefore, there exists a subsequence of $\left(\sigma^{n_{k}}\right)$ that converges to $\sigma \in \Sigma$. Rename this subsequence as $\left(\sigma^{n}\right)$ for notational simplicity. We have that $\sigma_{i}^{n}(a)(b, S) \rightarrow \sigma(i, a)(b, S)$ and that $f\left(x^{n}, \sigma^{n}\right) \rightarrow f(x, \sigma)$, because of the continuity of $y$, and thus $z=f(x, \sigma)$.

It is sufficient to show that $\sigma \in \Sigma(x)$. By the definition of $\Sigma(x), \sigma \in \Sigma(x)$ if and only if $\sigma \in \Sigma$ and $\sigma_{i}(a)(b, S)=0$ for all $(b, S)$ such that

$$
x_{S}(b)-x_{S}(a)<\max _{(b, S)}\left\{e(a)(S, b)(x): a \rightarrow_{S} b \text { and } i \in S\right\} .
$$

Consider any $S \subset \pi$ for which the inequality above holds. By continuity, we have that there exists a large enough $n_{0}$ such that, for all $n \geq n_{0}$,

$$
x_{S}^{n}(b)-x_{S}^{n}(a)<\max _{(b, S)}\left\{e(a)(S, b)\left(x^{n}\right): a \rightarrow_{S} b \text { and } i \in S\right\} .
$$

But since $\sigma^{n} \in \Sigma\left(x^{n}\right)$, this implies that $\sigma_{i}^{n}(a)(b, S)=0$ and $\sigma_{i}(a)(b, S)=0$.

Since all the conditions for the Kakutani fixed-point theorem hold, the correspondence $F$ has a fixed point, which yields an MPE.

We present below some other basic properties of the equilibrium. When we concatenate (1), (3), and (4), the expected payoffs $x_{i}(a)$ must satisfy the following system of equations:

$$
\begin{aligned}
x_{i}(a)= & (1-\delta) v_{i}(a)+\delta p_{i}(a) e_{i}(a)+\delta \sum_{j \in N} p_{j}(a) \sum_{(S, b)} \sigma_{j}(a)(S, b) \\
& \times\left[I(i \in S) x_{i}(a)+I(i \notin S) x_{i}(b)\right],
\end{aligned}
$$

where $e_{i}(a)$ is the gain or excess of proposer $i$ at state $a$ :

$$
e_{i}(a)=\max _{(b, S)}\left\{\sum_{j \in S}\left[x_{j}(b)-x_{j}(a)\right]: a \rightarrow_{S} b \text { and } i \in S\right\} .
$$

Our assumptions directly imply some other simple properties for the equilibrium. For example, by assumption 1 , any player can always decide to stay in the same state, and thus the excess is always positive. Moreover, by assumption 2, it must be that if player $i$ finds it optimal at state $a$ to approach coalition $S$ and propose a move to state $b$, that is, if $\sigma_{i}(a)(S, b)>0$, then agents outside $S$ should be no worse off in state $a$ than in state $b$, that is, $x_{j}(b) \leq x_{j}(a)$ for all $j \notin S$. QED

Proof of Voting Example

We rely on proposition 1 to construct the equilibrium. Consider the strategy profile below, where the payoffs $x_{i}(s)$ are given by equation (A3). At state $a$, player 1 proposes to player 3 to move to state $b$, offering him $x_{3}(a)$; player 2 proposes to player 3 to move to state $c$, offering him $x_{3}(a)$; and player 3 proposes to player 2 to move to state $c$, offering him $x_{2}(a)$ with probability $\mu$ and proposes to player 1 to move to state $a$, offering him $x_{1}(a)$ with probability $1-\mu$. Use symmetric strategies at the other states $b$ and $c$. At state $d, 1$ proposes to 2 to move to state $a$, offering $x_{2}(d) ; 2$ proposes to 3 to move to $c$, offering $x_{3}(d)$; and 3 proposes to 1 to move to $b$, offering $x_{1}(d)$.

Owing to the symmetry of the problem, we let $x_{1}=x_{1}(a)=x_{2}(c)=x_{3}(b)$, 
$x_{2}=x_{2}(a)=x_{1}(b)=x_{3}(c)$, and $x_{3}=x_{3}(a)=x_{2}(b)=x_{1}(c)$. Equations (A1) become

$$
\begin{aligned}
& x_{1}=(1-\delta) v_{1}(a)+\delta \frac{e}{3}+\frac{\delta}{3}\left[x_{1}+x_{3}+\mu x_{1}+(1-\mu) x_{3}\right], \\
& x_{2}=(1-\delta) v_{2}(a)+\delta \frac{e}{3}+\frac{\delta}{3}\left[x_{2}+x_{3}+\mu x_{3}+(1-\mu) x_{2}\right], \\
& x_{3}=(1-\delta) v_{3}(a)+\delta \frac{e}{3}+\frac{\delta}{3}\left(3 x_{3}\right),
\end{aligned}
$$

and the excess $e$ satisfies the equation

$$
\begin{aligned}
e & =\left(x_{1}+x_{2}\right)-\left(x_{1}+x_{3}\right) \\
& =\left(x_{1}+x_{2}\right)-\left(x_{2}+x_{3}\right)
\end{aligned}
$$

(player 3 is indifferent between moving to $b$ in coalition with 1 and moving to $c$ in coalition with 2). Solving for the system of equations yields the solution (for $\delta=0.95$ ) equal to $x_{1}=0.87, x_{2}=0.87, x_{3}=0.75, e=0.12$, and $\mu=$ 0.17 . The equilibrium at state $d$ is given by the solution to

$$
x=(1-\delta) v_{i}(d)+\delta\left[\frac{e^{\prime}}{3}+\frac{1}{3}\left(2 x+x_{3}\right)\right],
$$

where $e^{\prime}=\left(x_{1}+x_{2}\right)-2 x$; the solution is $x=0.87$ and $e^{\prime}=0.002$. It can be readily checked that there are no profitable deviations, and thus the payoff system satisfies condition ii of proposition 1, which proves that the strategy profile is an MPE. QED

\section{Proof of Proposition 2}

We first note that any spot contract can be represented, without any loss of generality, as a direct mechanism in which agents respond whether they accept or reject the spot contract. Of course, the contract needs to be feasible, that is, voluntary, and to satisfy the constraints imposed by the transition rule. Formally, we have the following definition.

Definition 4 . The offer $\tau$ by player $i$ at state $a$ is a feasible spot contract or mechanism if $\tau=(S, z, t)$, where $z: 2^{S} \rightarrow Z$ are response-contingent allocations and $t: 2^{S} \rightarrow R^{S}$ are response-contingent transfers, such that, for all subsets of players accepting the offer $A \subset S, a \rightarrow_{A} z(A)$ and $t_{j}(A)=0$ for all $j \notin S \backslash A$.

The extensive form of the dynamic economy in which general spot contracts are allowed is similar to the model in Section II, except for the more general offers. When the offer $\tau=(S, z, t)$ is made, the agents in $S$ respond to the offer by yes or no. ${ }^{47}$ If the offer is accepted by $A \subset S$, then the economy moves to the next period with state $z(A)$, and the (lump-sum) transfers $t(A)$ are made among agents in $A$. Of course, an agent who refuses the contract cannot be forced to pay a transfer; hence $t_{j}(A)=0$ for all $j \notin S \backslash A$.

Any MPE $\sigma$ of an economy with general spot contracts with continuation value

${ }^{47}$ To simplify the presentation, we may assume that agents respond simultaneously, and we restrict attention to equilibria in undominated strategies (to avoid coordination problems). 
$x_{i}(a)$ satisfies the following: proposer $i$ at state $a$ offers $\tau=(N, z, t)$ such that $z(N \backslash j) \in \arg \min \left\{x_{j}(\sigma \mid b): a \rightarrow_{N \backslash j} b\right\}$ and $z(N) \in \arg \max \left\{\sum_{j \in N} x_{j}(\sigma \mid b): a \rightarrow_{N} b\right\}$, and transfer $t_{j}(N)+x_{j}(\sigma \mid z(N))=x_{j}(\sigma \mid z(N \backslash j))$ for all $j \neq i$ and all responders accept offer $\tau$.

When player $i$ is the responder, his value is

$$
\phi_{i}^{R}(a)=\min \left\{x_{i}(b): a \rightarrow_{N \backslash i} b\right\}
$$

when he is the proposer, his value is

$$
\phi_{i}^{P}(a)=\max \left\{\sum_{j \in N} x_{j}(b): a \rightarrow_{N} b\right\}-\sum_{j \neq i} \min \left\{x_{j}(b): a \rightarrow_{N \backslash j} b\right\} .
$$

Moreover, the equilibrium value satisfies $\phi_{i}(a)=p_{i}(a) \phi_{i}^{P}(a)+\left[1-p_{i}(a)\right] \phi_{i}^{R}(a)$. Concatenating these expressions implies that the expected payoffs $x_{i}(a)$ must satisfy the following system of equations:

$$
\begin{aligned}
x_{i}(a)= & (1-\delta) v_{i}(a)+\delta \min \left\{x_{i}(b): a \rightarrow_{N \backslash i} b\right\} \\
& +\delta p_{i}(a)\left(\max \left\{x_{N}(b): a \rightarrow_{N} b\right\}-\sum_{j \in N} \min \left\{x_{j}(b): a \rightarrow_{N \backslash j} b\right\}\right) .
\end{aligned}
$$

The maximum from any state $a, \max \left\{x_{N}(b): a \rightarrow_{N} b\right\}$, satisfies $\max \left\{x_{N}(b)\right.$ : $\left.a \rightarrow_{N} b\right\}=\max \left\{x_{N}(a): a \in Z\right\}$ and does not depend on the initial state, because by assumption 3 , we must have $Z=\left\{b \in Z, a \rightarrow_{N} b\right\}$. Let $x^{*}=\max \left\{x_{N}(a): a \in\right.$ $Z$ \}. Adding up all the equations (A4) above yields that the aggregate value is equal to $x_{N}(a)=(1-\delta) v_{N}(a)+\delta x^{*}$. But then

$$
x^{*}=\max \left\{x_{N}(a): a \in Z\right\}=(1-\delta) \max \left\{v_{N}(a): a \in Z\right\}+\delta x^{*},
$$

which implies that $x^{*}=\max \left\{v_{N}(a): a \in Z\right\}$.

The strategy of players is, from any state, to move to the state that maximizes $\max \left\{x_{N}(a): a \in Z\right\}$. But since this maximum attains the efficient amount $\max \left\{v_{N}(a): a \in Z\right\}$, this implies that, from any state, we move to the efficient allocation. QED

Proof of Proposition 3

Given an MPE $\sigma$ of the simple-offer contract game, define the following strategy profile $\sigma_{c}$ of the spot contract + collusion game: proposers make an offer $\tau=$ $(S, z, t)$ with $z(S)=b$ and $t_{j}(S)=x_{j}(\sigma \mid a)-x_{j}(\sigma \mid b)$, and $z(A)=a$ and $t_{j}(A)=0$ for all $A \neq S$ with probability $\sigma_{i}(a)(S, b)>0$; given any offer $\tau=(S, z, t)$, the third party coordinates the acceptance by the subset $A \subset S$ that maximizes $\max _{A \subset s}\left\{\sum_{j \in S \backslash i\}} x_{j}(\sigma \mid z(A))+t_{j}(z(A))\right\}$.

By construction, the strategy $\sigma_{c}$ is equivalent to the strategy $\sigma$ (the continuation values $x_{j}(\sigma \mid a)=x_{j}\left(\sigma_{c} \mid a\right)=x_{j}(a)$ and transitions induced by both strategies are the same). So it remains to show that $\sigma_{c}$ is an MPE: (i) The proposer $i$ cannot do strictly better. Indeed the possibility of collusion means that by all refusing the contract, the set of approached agents $S \backslash\{i\}$ can always guarantee a payoff equal to $\sum_{j \in S \backslash i\}} x_{j}(a)$ since $i$ would then be forced to stay in state $a$, by assumption 4. So if all agents $S$ agree to the contract specifying a move to, say, state $b$, where $a \rightarrow_{S} b$, then $i$ 's payoff must be bounded by $\sum_{j \in S} x_{j}(b)-\sum_{j \in S \backslash i\}} x_{j}(a)$. But this is exactly the payoff that $i$ gets when offering $\tau=(S, z, t)$. (ii) The third-party 
strategy, by definition, is a best response. So this proves that $\sigma_{c}$ is an equivalent MPE. The converse part follows along the same line.

To simplify the exposition, we have assumed that the collusive ring could be organized by a third benevolent party. Note that our insight would carry over if the collusive ring were instead organized by third parties interested in making money, and some competition among third parties would allow the ring to retain some of the surplus. (The proof above shows that the proposer is best off by offering contracts that do not allow the approached members to collude. This conclusion would hold true also under the alternative specification just suggested.) QED

Proof of Proposition 4

Suppose by contradiction that there exists a subsequence $\delta_{n} \rightarrow 1$ such that

$$
\lim _{\delta_{n} \rightarrow 1} \max \left\{\left|\phi_{N}^{\left(\delta_{n}\right)}(a)-\phi_{N}^{\left(\delta_{n}\right)}(b)\right|: a, b \in Z\right\}>0,
$$

where $\mu^{\left(\delta_{n}\right)}$ and $\phi^{\left(\delta_{n}\right)}$ are the equilibrium transition probabilities and payoffs. Now consider a convergent subsequence of $\delta_{n}$ such that $\mu^{\left(\delta_{n}\right)} \rightarrow \mu$ and $\phi^{\left(\delta_{n}\right)} \rightarrow \phi$ (of course, $\left.\max _{a, b \in Z}\left|\phi_{N}(a)-\phi_{N}(b)\right|>0\right)$.

The aggregate value $\phi_{N}^{(\delta)}(\cdot)$ satisfies

$$
\phi_{N}^{(\delta)}(a)=\sum_{b} \mu^{(\delta)}(a, b)\left[(1-\delta) v_{N}(b)+\delta \phi_{N}^{(\delta)}(b)\right] \quad \text { for all } a \in Z,
$$

which is equivalent to (in matrix representation)

$$
\left[I-\delta \mu^{(\delta)}\right] \phi_{N}^{(\delta)}=(1-\delta) \mu^{(\delta)} v_{N}
$$

Taking the limit when $\delta \rightarrow 1$, we have

$$
(I-\mu) \phi_{N}=0 .
$$

Let $E_{1}, \ldots, E_{m}$ be the ergodic classes and $T \subset Z$ the class of transient states of the limit transition probability $\mu\left(Z=E_{1} \cup \cdots \cup E_{m} \cup T\right)$. Equation (A6) is equivalent to $\phi_{N}=\mu \phi_{N}$, and thus $\phi_{N}$ is a (right) eigenvector of $\mu$ corresponding to the eigenvalue one. A well-known result from the theory of Markov chains (see Doob 1953) implies that $\phi_{N}(\cdot)$ is a constant within each ergodic class $E_{j}$ and that the value of $\phi_{N}(\cdot)$ at any transient state is a linear combination of the values of $\phi_{N}(\cdot)$ at the ergodic states.

LEMma 1. Consider an equilibrium strategy profile $\sigma^{(\delta)}$ of $\mathcal{E}(\rightarrow, v, p, \delta)$ such that the equilibrium transition probabilities and payoffs $\mu^{(\delta)}$ and $\phi^{(\delta)}$ are such that $\mu^{(\delta)} \rightarrow \mu$ and $\phi^{(\delta)} \rightarrow \phi$, as $\delta \rightarrow 1$, and let $E \subset Z$ be a stable set of $\mu$. Consider a player $i$ who can be the proposer with positive probability at some state in $E$. Then there exists a state $a^{*} \in E$ such that $p_{i}\left(a^{*}\right)>0$ and $i$ 's excess at $a^{*}$ converges to zero, that is, $\lim _{\delta \rightarrow 1} e_{i}^{(\delta)}\left(a^{*}\right)=0$.

Proof of lemma 1. Let $e_{i}(a)=\lim _{\delta \rightarrow 1} e_{i}^{(\delta)}(a)$. Taking the limit of the expressions in equation (A1), we have that

$$
x_{i}(a)=p_{i}(a) e_{i}(a)+\sum_{(S, b)} \mu(a, b, S)\left[I(i \in S) x_{i}(a)+I(i \notin S) x_{i}(b)\right],
$$

where $\mu(a, b, S)=\sum_{j \in N} p_{j}(a) \sigma_{j}(a)(S, b)$. We have already seen that $x_{i}(b) \leq x_{i}(a)$ for all $b \in Z$ such that $\mu(a, b, S)>0$ and $i \notin S$ (see the remarks at the end of the proof of proposition 1).

Let player $i$ be a proposer with positive probability in at least one state in $E$ 
(i.e, $p_{i}\left(a^{\prime}\right)>0$ for some $a^{\prime} \in E$ ). Let $x$ be the limit solution and $\mu$ the limit transition probability. For any player $i$, let $a$ be a state in which $\min _{a^{\prime} \in E}\left\{x_{i}\left(a^{\prime}\right)\right\}$ is attained $\left(x_{i}(a)=\min _{a^{\prime} \in E}\left\{x_{i}\left(a^{\prime}\right)\right\}\right)$, and thus $\mu(a, b, S)>0$ implies that $x_{i}(b) \geq$ $x_{i}(a)$ (because $E$ is a closed class, and $\mu(a, b, S)>0$ and $a \in E$ implies that $b \in$ E).

Suppose that $p_{i}(a)>0$. Applying equation (A7) to state $a$ and taking into account that $\sum_{(S, b)} \mu(a, b, S)=1$, we get that

$$
x_{i}(a)=p_{i}(a) e_{i}(a)+x_{i}(a),
$$

which implies $e_{i}(a)=0$, since $p_{i}(a)>0$.

Now if $p_{i}(a)=0$, then any state $b$ such that $\mu(a, b, S)>0$ is also such that $x_{i}(b)=\min _{a^{\prime} \in E}\left\{x_{i}\left(a^{\prime}\right)\right\}$ (this is so because $x_{i}(b) \leq x_{i}(a)$ for all $b \in Z$ such that $\mu(a, b, S)>0)$. Thus one can find a state $a^{*}$ such that $x_{i}\left(a^{*}\right)=\min _{a^{\prime} \in E}\left\{x_{i}\left(a^{\prime}\right)\right\}$ and $p_{i}\left(a^{*}\right)>0$ and use the same argument to conclude this state. QED

So by lemma 1 there a exists state $a_{j}$ in each ergodic class $E_{j}$ such that the excess $e_{i}^{\left(\delta_{n}\right)}\left(a_{j}\right)$ of player $i$ at state $a_{j}$ converges to zero: $e_{i}^{\left(\delta_{n}\right)}\left(a_{j}\right) \rightarrow 0$, for all $j=$ $1, \ldots, m$.

We now show that the values of $\phi_{N}(\cdot)$ across ergodic classes are equal: say that there are two ergodic classes $E_{j}$ and $E_{k}$ such that $\phi_{N}\left(a_{j}\right)<\phi_{N}\left(a_{k}\right)$. But since it is feasible for player $i$ to move from state $a_{j}$ to state $a_{k}$ with the agreement of all players $N, \lim _{\delta_{n} \rightarrow 1} \sup e_{i}^{\left(\delta_{n}\right)}\left(a_{j}\right) \geq \phi_{N}\left(a_{k}\right)-\phi_{N}\left(a_{j}\right)>0$ (a contradiction). Also, because the value of $\phi_{N}(\cdot)$ at transient states is a linear combination of the values at ergodic states, we conclude that $\phi_{N}(\cdot)$ is constant across all states in Z. Finally, this leads to a contradiction with $\max _{a, b \in Z}\left|\phi_{N}(a)-\phi_{N}(b)\right|>0$, completing the proof. QED

\section{Proof of Proposition 5}

Suppose by contradiction that there is a sequence of $\delta_{n}$ converging to one $\left(\delta_{n} \rightarrow 1\right)$ with equilibrium transition probability $\mu^{(\delta)}$ and payoff $\phi^{(\delta)}$ having two ergodic classes $E_{1}^{(\delta)}$ and $E_{2}^{(\delta)}$ with invariant probabilities $\lambda_{1}^{(\delta)}$ and $\lambda_{2}^{(\delta)}$ (given by $\lambda_{i}^{(\delta)} \mu^{(\delta)}=\lambda_{i}^{(\delta)}$ and $\left.\sum_{a^{\prime} \in E_{i}^{(\delta)}} \lambda_{i}^{(\delta)}\left(a^{\prime}\right)=1\right)$. Consider a convergent subsequence of $\delta_{n}$ (also named $\delta_{n}$ ) such that $\mu^{\left(\delta_{n}\right)} \rightarrow \mu, \phi^{\left(\delta_{n}\right)} \rightarrow \phi, \lambda_{i}^{\left(\delta_{n}\right)} \rightarrow \lambda_{i}$, and $E_{i}=E_{i}^{\left(\delta_{n}\right)}$.

Multiplying equation (A5) to the left by $\lambda_{i}^{(\delta)}$ yields

$$
\lambda_{i}^{(\delta)}\left[I-\delta \mu^{(\delta)}\right] \phi_{N}^{(\delta)}=\lambda_{i}^{(\delta)}(1-\delta) \mu^{(\delta)} v_{N}
$$

which is equivalent to $(1-\delta) \lambda_{i}^{(\delta)} \phi_{N}^{(\delta)}=\lambda_{i}^{(\delta)}(1-\delta) \mu^{(\delta)} v_{N}$ (after taking into account that $\left.\lambda_{i}^{(\delta)} \mu^{(\delta)}=\lambda_{i}^{(\delta)}\right)$, and thus

$$
\lambda_{i}^{(\delta)} \phi_{N}^{(\delta)}=\lambda_{i}^{(\delta)} \mu^{(\delta)} v_{N}
$$

Taking the limit of the above expression (using $\lambda_{i} \mu=\lambda_{i}$ ), we have

$$
\lambda_{i} \phi_{N}=\lambda_{i} \mu v_{N}=\lambda_{i} v_{N}
$$

But proposition 4 implies that $\phi_{N}(\cdot)$ is constant over $Z$, and thus

$$
\lambda_{1} v_{N}=\sum_{a^{\prime} \in E_{2}} \lambda_{i}\left(a^{\prime}\right) v_{N}\left(a^{\prime}\right)=\sum_{a^{\prime} \in E_{2}} \lambda_{i}\left(a^{\prime}\right) v_{N}\left(a^{\prime}\right)=\lambda_{2} v_{N} .
$$

Finally, the equality $\lambda_{1} v_{N}=\lambda_{2} v_{N}$ cannot be satisfied generically: The two ergodic classes are disjoint, $E_{1} \cap E_{2}=\varnothing$, and the invariant measures $\lambda_{i}$ depend on only the payoffs $v(a)$ for $a \in E_{i}$. If equality (A10) happened to be satisfied for some choice of parameters, changing slightly the payoffs in one of the classes 
(say by adding an $\epsilon$ to the payoff of a player in one of the classes) would lead to violation of the equality. QED

\section{Proof of Proposition 6}

Stability: Let $a^{*} \in \mathrm{ENF}$. We claim that $\phi_{i}\left(a^{*}\right)=v_{i}\left(a^{*}\right)$ for all $i \in N$. Indeed any player $i$ can get a utility level at least equal to $v_{i}\left(a^{*}\right)$ if he does not make any proposals and if he does not accept any proposals (whenever $a^{*} \rightarrow_{S_{1}} \cdots \rightarrow_{S_{k}} a_{k} \rightarrow$ $\cdots \rightarrow_{S_{n}} a_{n}$ and $\left.i \notin S_{k}, v_{i}\left(a_{n}\right) \geq v_{i}\left(a^{*}\right)\right)$. Therefore, $\phi_{i}\left(a^{*}\right) \geq v_{i}\left(a^{*}\right)$. But $a^{*}$ is also efficient, and so $v_{N}\left(a^{*}\right)=\max _{a \in Z} v_{N}(a)$, which together imply $\phi_{i}\left(a^{*}\right)=v_{i}\left(a^{*}\right)$.

We now show that any move $a^{*} \rightarrow_{S} b$ by coalition $S$ yields a nonpositive excess, where the excess associated with the move is $e=x_{S}(b)-x_{S}\left(a^{*}\right)$, which is sufficient to prove that $a$ is a stable state.

Efficiency of $a^{*}$ implies that $v_{N}\left(a^{*}\right) \geq v_{N}(b)$ and $v_{N}\left(a^{*}\right) \geq \phi_{N}(b)$ for all $b \in Z$. Moreover, if $a^{*} \rightarrow_{S} b$, then $\phi_{N \mid S}(b) \geq v_{N \mid S}\left(a^{*}\right)$ (this comes from the definition of $\mathrm{ENF})$. Concatenating the inequalities yields that

$$
e=x_{S}(b)-x_{S}\left(a^{*}\right)=\delta\left[\phi_{S}(b)-\phi_{S}\left(a^{*}\right)\right]+(1-\delta)\left[v_{S}(b)-v_{S}\left(a^{*}\right)\right] \leq 0
$$

because

$$
\begin{aligned}
\phi_{S}(b)-\phi_{S}\left(a^{*}\right) & \leq\left[\phi_{S}(b)-\phi_{S}\left(a^{*}\right)\right]+\left[\phi_{N \mid S}(b)-v_{N \mid S}\left(a^{*}\right)\right] \\
& =\phi_{N}(b)-v_{N}\left(a^{*}\right) \leq 0,
\end{aligned}
$$

and also it is easy to see that $v_{s}(b)-v_{s}(a) \leq 0$.

Efficiency: Let $a^{*}$ be an absorbing state. Then $e_{i}\left(a^{*}\right)=0$ for all $i$, and because $\mu\left(a^{*}, a^{*}\right)=1$, equation (A1) corresponds to

$$
x_{i}\left(a^{*}\right)=\delta x_{i}\left(a^{*}\right)+(1-\delta) v_{i}\left(a^{*}\right) .
$$

Thus $x_{i}\left(a^{*}\right)=v_{i}\left(a^{*}\right)$, and $\phi_{N}\left(a^{*}\right)=x_{N}\left(a^{*}\right)=v_{N}\left(a^{*}\right)$. Since $a^{*}$ is an efficient state, $\phi_{N}\left(a^{*}\right)=\max _{a \in Z} v_{N}(a)$. The result now follows from proposition 4. QED

\section{Proof of Proposition 7}

Stability: Consider any state $a$ that is not negative externality-free. By definition, there exists a sequence $a=a_{0} \rightarrow_{N \backslash i} a_{1} \rightarrow \cdots \rightarrow a_{q-1} \rightarrow_{N \backslash i} a_{q}=b$ of moves such that $v_{i}(b)<v_{i}(a)$. Consider now a proposer probability $p$ satisfying (or approximately satisfying) $p_{j}\left(a_{k}\right)=1$ for some $j \in N \backslash i$ and all $k=1, \ldots, q-1$, and $p_{i}(b)=0$.

Suppose by contradiction that there exists a sequence of equilibria $\sigma^{(\delta)}$ of $\mathcal{E}(\rightarrow$, $v, p, \delta$ ) with $\delta$ converging to one, such that $\phi^{(\delta)} \rightarrow \phi\left(\right.$ and $\left.x^{(\delta)} \rightarrow x\right)$ and state $a$ is a stable state for any $\delta$ in the sequence. We claim that this is not possible.

The stability of $a$ implies that $\phi_{l}(a)=v_{l}(a)$ for all $l \in N$ (and $x=\lim _{\delta \rightarrow 1} x^{(\delta)}$ satisfies $x=\phi$ ). Consider the following deviation from the equilibrium strategy by player $j$ : propose to move $a_{k} \rightarrow_{N \backslash i} a_{k+1}$ for all $k=0, \ldots, q-1$ offering each player $l \in N \backslash\{i, j\}$ a transfer $t_{l}$ such that $t_{l}+x_{l}\left(a_{k+1}\right)=x_{l}\left(a_{k}\right)+\epsilon$, where $\epsilon>0$. The best-response strategy of all players $l$ is to accept such offers. The deviation produces a (limit) gain to player $j$, with respect to the no-deviation strategy, of $G=\left[x_{N \mid i}(b)-x_{N \mid i}(a)\right]+O(1-\delta, \epsilon)$. But because player $i$ is not a proposer at $b$, $i$ 's excess at $b$ is zero, and equation (A1) implies that $\phi_{i}(b) \leq v_{i}(b)$. Moreover, proposition 4 implies that $v_{N}(a)=\phi_{N}(a)=\phi_{N}(b)$, and thus $G=\phi_{N}(b)-$ $\phi_{N}(b)+x_{i}(a)-x_{i}(b)+O(1-\delta, \epsilon)$ is no smaller than $v_{i}(a)-v_{i}(b)>0$ for $\delta$ close enough to zero and $\epsilon$ sufficiently small, which leads to a contradiction. 
Efficiency: Consider any small perturbation $v^{\prime}$ of the payoff $v$ such that the new payoff $v^{\prime}$ has only one efficient state, say $a$. Of course, for any small enough perturbation, $a$ is also an efficient state of $E(v)$, and since $a \notin \operatorname{ENF}(v)$, there must exist a player $i$ and a sequence $a=a_{0} \rightarrow_{N \backslash i} a_{1} \rightarrow \cdots \rightarrow a_{q-1} \rightarrow_{N \backslash i} a_{q}=b$ of moves such that $v_{i}(b)<v_{i}(a)$. Note that any small perturbation also satisfies $v_{i}^{\prime}(b)<v_{i}^{\prime}(a)$ and $v_{N}^{\prime}(b)<v_{N}^{\prime}(a)$ ( $a$ is the only efficient state of $\mathcal{E}\left(\rightarrow, v^{\prime}\right)$ ). Consider now any proposer probability $p$ chosen as in the previous paragraph.

We claim that economy $\mathcal{E}\left(\rightarrow, v^{\prime}, p\right)$ is not asymptotically efficient. Suppose to the contrary that it is asymptotically efficient, so that for any sequence $\sigma^{(\delta)}$ with $\phi^{(\delta)} \rightarrow \phi$ and $\mu^{(\delta)} \rightarrow \mu$ the payoffs satisfy $\phi_{N}(\cdot)=\phi_{N}(a)=v_{N}^{\prime}(a)=\max _{a^{\prime} \in Z} v_{N}^{\prime}\left(a^{\prime}\right)$. Since $a$ is the only efficient state of $\mathcal{E}\left(\rightarrow, v^{\prime}\right), \mu(a, a)=1$ and $\phi_{l}(a)=v_{l}^{\prime}(a)$ for all $l \in N$. An argument similar to the one used above (applied to state $a$, which is also not negative externality-free) now yields a contradiction. QED

\section{References}

Banks, Jeffrey, and John Duggan. 2001. "A Bargaining Model of Legislative PolicyMaking.” Manuscript, Univ. Rochester.

Baron, David P., and John A. Ferejohn. 1989. "Bargaining in Legislatures." American Polit. Sci. Rev. 83 (December): 1181-1206.

Binmore, Ken, Ariel Rubinstein, and Asher Wolinsky. 1986. "The Nash Bargaining Solution in Economic Modelling." Rand J. Econ. 17 (Summer): 176-88.

Bloch, Francis. 1996. "Sequential Formation of Coalitions in Games with Externalities and Fixed Payoff Division." Games and Econ. Behavior 14 (May): 90123.

Caillaud, Bernard, and Philippe Jehiel. 1998. "Collusion in Auctions with Externalities." Rand J. Econ. 29 (Winter): 680-702.

Chatterjee, Kalyan, Bhaskar Dutta, Debraj Ray, and Kunal Sengupta. 1993. "A Noncooperative Theory of Coalitional Bargaining." Rev. Econ. Studies 60 (April): 463-77.

Chwe, Michael S. 1994. "Farsighted Coalitional Stability." J. Econ. Theory 63 (August): 299-325.

Coase, Ronald H. 1960. "The Problem of Social Cost." J. Law and Econ. 3 (October): 1-44.

Diermeier, Daniel, and Antonio Merlo. 2000. "Government Turnover in Parliamentary Democracies." J. Econ. Theory 94 (September): 46-79.

Diermeier, Daniel, and Roger B. Myerson. 1999. "Bicameralism and Its Consequences for the Internal Organization of Legislatures." A.E.R. 89 (December): 1182-96.

Doob, Joseph L. 1953. Stochastic Processes. New York: Wiley.

Fudenberg, Drew, and Jean Tirole. 1991. Game Theory. Cambridge, MA: MIT Press.

Gale, Douglas M. 1986. "Bargaining and Competition Part I: Characterization." Econometrica 54 (July): 785-806.

Gomes, Armando. Forthcoming. "Multilateral Contracting with Externalities." Econometrica.

Graham, Daniel A., and Robert C. Marshall. 1987. "Collusive Bidder Behavior at Single-Object Second-Price and English Auctions.” J.P.E. 95 (December): 1217-39.

Greenberg, Joseph. 1990. The Theory of Social Situations: An Alternative GameTheoretic Approach. Cambridge: Cambridge Univ. Press. 
Gul, Faruk. 1989. "Bargaining Foundations of Shapley Value." Econometrica 57 (January): 81-95.

Hart, Sergiu, and Andreu Mas-Colell. 1996. "Bargaining and Value." Econometrica 64 (March): 357-80.

Jehiel, Philippe, and Benny Moldovanu. 1995a. "Cyclical Delay in Bargaining with Externalities.” Rev. Econ. Studies 62 (October): 619-37.

— 1995b. "Negative Externalities May Cause Delay in Negotiation." Econometrica 63 (November): 1321-35.

- 1999. "Resale Markets and the Assignment of Property Rights." Rev. Econ. Studies 66 (October): 971-91.

Jehiel, Philippe, Benny Moldovanu, and Ennio Stacchetti. 1996. "How (Not) to Sell Nuclear Weapons.” A.E.R. 86 (September): 814-29.

Konishi, Hideo, and Debraj Ray. 2003. "Coalition Formation as a Dynamic Process." J. Econ. Theory 110 (May): 1-41.

Laffont, Jean-Jacques, and David Martimort. 1997. "Collusion under Asymmetric Information.” Econometrica 65 (July): 875-911.

McAfee, R. Preston, and John McMillan. 1992. "Bidding Rings." A.E.R. 82 (June): 579-99.

Moldovanu, Benny, and Eyal Winter. 1995. "Order Independent Equilibria." Games and Econ. Behavior 9 (April): 21-34.

Perry, Motty, and Philip J. Reny. 1994. "A Noncooperative View of Coalition Formation and the Core." Econometrica 62 (July): 795-817.

Ray, Debraj, and Rajiv Vohra. 1999. "A Theory of Endogenous Coalition Structures." Games and Econ. Behavior 26 (February): 286-336.

Rosenthal, Robert W. 1972. "Cooperative Games in Effectiveness Form." J. Econ. Theory 5 (August): 88-101.

Rubinstein, Ariel, and Asher Wolinsky. 1985. "Equilibrium in a Market with Sequential Bargaining." Econometrica 53 (September): 1133-50.

Segal, Ilya. 1999. "Contracting with Externalities." Q.J.E. 114 (May): 337-88.

Shepsle, Kenneth A. 1979. "Institutional Arrangements and Equilibrium in Multidimensional Voting Models." American J. Polit. Sci. 23 (February): 27-59.

Xue, Licun. 1998. "Coalitional Stability under Perfect Foresight." Econ. Theory 11 (May): 603-27. 